\title{
Pacific
}

Journal of

Mathematics

\section{A COMPACT EMBEDDING THEOREM FOR GENERALIZED SOBOLEV SPACES}

Seng-Kee Chua, Scott Rodney And Richard L. Wheeden 


\title{
A COMPACT EMBEDDING THEOREM FOR GENERALIZED SOBOLEV SPACES
}

\author{
Seng-Kee Chua, Scott Rodney And Richard L. WheEden
}

\begin{abstract}
We give an elementary proof of a compact embedding theorem in abstract Sobolev spaces. The result is first presented in a general context and later specialized to the case of degenerate Sobolev spaces defined with respect to nonnegative quadratic forms on $\mathbb{R}^{n}$. Although our primary interest concerns degenerate quadratic forms, our result also applies to nondegenerate cases, and we consider several such applications, including the classical Rellich-Kondrachov compact embedding theorem and results for the class of $s$-John domains in $\mathbb{R}^{n}$, the latter for weights equal to powers of the distance to the boundary. We also derive a compactness result for Lebesgue spaces on quasimetric spaces unrelated to $\mathbb{R}^{n}$ and possibly without any notion of gradient.
\end{abstract}

\section{The general theorem}

The main goal of this paper is to generalize the classical Rellich-Kondrachov theorem concerning compact embedding of Sobolev spaces into Lebesgue spaces. Our principal result applies not only to the classical Sobolev spaces on open sets $\Omega \subset \mathbb{R}^{n}$ but also allows us to treat the degenerate Sobolev spaces defined in [Sawyer and Wheeden 2010] and to obtain compact embedding of them into various $L^{q}(\Omega)$ spaces. These degenerate Sobolev spaces are associated with quadratic forms $Q(x, \xi)=\xi^{\prime} Q(x) \xi, x \in \Omega, \xi \in \mathbb{R}^{n}$, which are nonnegative but may vanish identically in $\xi$ for some values of $x$. Such quadratic forms and Sobolev spaces arise naturally in the study of existence and regularity of weak solutions of some second order subelliptic linear/quasilinear partial differential equations; see, for example, [Sawyer and Wheeden 2006; Rodney 2007; 2012; Monticelli et al. 2012; Rios et al. 2013].

The Rellich-Kondrachov theorem is frequently used to study the existence of solutions to elliptic equations, a famous example being subcritical and critical

Chua was partially supported by Singapore Ministry of Education Academic Research Fund Tier 1 R146-000-150-112. Rodney was partially supported by the Natural Sciences and Engineering Research Council, Canada.

MSC2010: primary 46B50, 46E35; secondary 35H20.

Keywords: compact embedding, Sobolev spaces, degenerate quadratic forms. 
Yamabe equations, resulting in the solution of Yamabe's problem; see [Yamabe 1960; Trudinger 1968; Aubin 1976; Schoen 1984]. Further applications lie in proving the existence of weak solutions to Dirichlet problems for elliptic equations with rough boundary data and coefficients; see [Gilbarg and Trudinger 1997]. In a sequel to this paper, we will apply our compact embedding results to study the existence of solutions for some classes of degenerate equations.

In this section, we state and prove our most general compact embedding results. In Sections 2 and 3, we study some applications to classical and degenerate Sobolev spaces, respectively. In Section 4, more general results in quasimetric spaces are studied.

We begin by listing some useful notation. Let $w$ be a measure on a $\sigma$-algebra $\Sigma$ of subsets of a set $\Omega$, with $\Omega \in \Sigma$. For $0<p \leq \infty$, let $L_{w}^{p}(\Omega)$ denote the class of real-valued measurable functions $f$ satisfying $\|f\|_{L_{w}^{p}(\Omega)}<\infty$, where $\|f\|_{L_{w}^{p}(\Omega)}=$ $\left(\int_{\Omega}|f|^{p} d w\right)^{1 / p}$ if $p<\infty$ and $\|f\|_{L_{w}^{\infty}(\Omega)}=\operatorname{ess~sup~}_{\Omega}|f|$, the essential supremum being taken with respect to $w$-measure. When dealing with generic functions in $L_{w}^{p}(\Omega)$, we will not distinguish between functions which are equal a.e.- $w$. For $E \in \Sigma, w(E)$ denotes the $w$-measure of $E$, and if $0<w(E)<\infty, f_{E, w}$ denotes the $w$-average of $f$ over $E: f_{E, w}=\int_{E} f d w / w(E)$. Throughout the paper, positive constants are denoted by $C$ or $c$ and their dependence on important parameters is indicated.

For $k \in \mathbb{N}$, let $\mathscr{L}(\Omega)$ be a normed linear space of measurable $\mathbb{R}^{k}$-valued functions $\boldsymbol{g}$ defined on $\Omega$ with norm $\|\boldsymbol{g}\|_{\mathscr{L}(\Omega)}$. We assume that there is a subset $\Sigma_{0} \subset \Sigma$ such that $\left(\mathscr{L}(\Omega), \Sigma_{0}\right)$ satisfies the following properties:

(A) For any $\boldsymbol{g} \in \mathscr{L}(\Omega)$ and $F \in \Sigma_{0}$, the function $\boldsymbol{g} \chi_{F} \in \mathscr{X}(\Omega)$, where $\chi_{F}$ denotes the characteristic function of $F$.

$\left(\mathrm{B}_{p}\right)$ There are constants $C_{1}, C_{2}, p$ satisfying $1 \leq C_{1}, C_{2}, p<\infty$ and such that if $\left\{F_{l}\right\}$ is a finite collection of sets in $\Sigma_{0}$ with $\sum_{l} \chi_{F_{l}}(x) \leq C_{1}$ for all $x \in \Omega$, then

$$
\sum_{l}\left\|\boldsymbol{g} \chi_{F_{l}}\right\|_{\mathscr{L}(\Omega)}^{p} \leq C_{2}\|\boldsymbol{g}\|_{\mathscr{L}(\Omega)}^{p} \quad \text { for all } \boldsymbol{g} \in \mathscr{X}(\Omega)
$$

For $1 \leq N \leq \infty$, we will often consider the product space $L_{w}^{N}(\Omega) \times \mathscr{X}(\Omega)$. This is a normed linear space with norm

$$
\|(f, \boldsymbol{g})\|_{L_{w}^{N}(\Omega) \times \mathscr{L}(\Omega)}=\|f\|_{L_{w}^{N}(\Omega)}+\|\boldsymbol{g}\|_{\mathscr{L}(\Omega)} .
$$

A set $\mathscr{S} \subset L_{w}^{N}(\Omega) \times \mathscr{X}(\Omega)$ will be called a bounded set in $L_{w}^{N}(\Omega) \times \mathscr{X}(\Omega)$ if

$$
\sup _{(f, \boldsymbol{g}) \in \mathscr{S}}\|(f, \boldsymbol{g})\|_{L_{w}^{N}(\Omega) \times \mathscr{L}(\Omega)}<\infty
$$


Projection maps such as the one defined by

$$
\pi:(f, \boldsymbol{g}) \rightarrow f, \quad(f, \boldsymbol{g}) \in L_{w}^{N}(\Omega) \times \mathscr{X}(\Omega),
$$

play a role in our results. If $w(\Omega)<\infty, \pi\left(L_{w}^{N}(\Omega) \times \mathscr{X}(\Omega)\right) \subset L_{w}^{q}(\Omega)$ if $1 \leq q \leq N$.

Theorem 1.1. Let $w$ be a finite measure on a $\sigma$-algebra $\Sigma$ of subsets of a set $\Omega$, with $\Omega \in \Sigma$. Let $1 \leq p<\infty, 1<N \leq \infty, \mathscr{X}(\Omega)$ be a normed linear space satisfying properties $(\mathrm{A})$ and $\left(\mathrm{B}_{p}\right)$ relative to a collection $\Sigma_{0} \subset \Sigma$, and let $\mathscr{Y}$ be a bounded set in $L_{w}^{N}(\Omega) \times \mathscr{X}(\Omega)$.

Suppose that $\mathscr{Y}$ satisfies the following: given $\epsilon>0$, there are a finite number of pairs $\left\{E_{l}, F_{l}\right\}_{l=1}^{J}$ with $E_{l} \in \Sigma$ and $F_{l} \in \Sigma_{0}$ (the pairs and $J$ may depend on $\epsilon$ ) satisfying these properties:

(i) $w\left(\Omega \backslash \bigcup_{l} E_{l}\right)<\epsilon$ and $w\left(E_{l}\right)>0$.

(ii) $\left\{F_{l}\right\}$ has bounded overlaps independent of $\epsilon$ with the same overlap constant as in $\left(\mathrm{B}_{p}\right)$, that is,

$$
\sum_{l=1}^{J} \chi_{F_{l}}(x) \leq C_{1}, \quad x \in \Omega,
$$

for $C_{1}$ as in $\left(\mathrm{B}_{p}\right)$.

(iii) For every $(f, \boldsymbol{g}) \in \mathscr{Y}$, the local Poincaré-type inequality

$$
\left\|f-f_{E_{l}, w}\right\|_{L_{w}^{p}\left(E_{l}\right)} \leq \epsilon\left\|\boldsymbol{g} \chi_{F_{l}}\right\|_{\mathscr{L}(\Omega)}
$$

holds for each $\left(E_{l}, F_{l}\right)$.

Let $\hat{\mathscr{S}}$ be the set defined by

(1-5) $\hat{\mathscr{Y}}=\left\{f \in L_{w}^{N}(\Omega)\right.$ : there exists $\left\{\left(f^{j}, \boldsymbol{g}^{j}\right)\right\}_{j=1}^{\infty} \subset \mathscr{Y}$ with $f^{j} \rightarrow f$ a.e. $\left.-w\right\}$.

Then $\hat{\mathscr{S}}$ is compactly embedded in $L_{w}^{q}(\Omega)$ if $1 \leq q<N$ in the sense that, for every sequence $\left\{f_{k}\right\} \subset \hat{\mathscr{Y}}$, there is a single subsequence $\left\{f_{k_{i}}\right\}$ and a function $f \in L_{w}^{N}(\Omega)$ such that $f_{k_{i}} \rightarrow f$ pointwise a.e.-w in $\Omega$ and in $L_{w}^{q}(\Omega)$ norm for $1 \leq q<N$.

Before proceeding with the proof of Theorem 1.1, we make several simple observations. First, in the definition of $\hat{\mathscr{Y}}$, the property that $f \in L_{w}^{N}(\Omega)$ follows by Fatou's lemma since the associated functions $f^{j}$ are bounded in $L_{w}^{N}(\Omega)$, as $\mathscr{S}$ is bounded in $L_{w}^{N}(\Omega) \times \mathscr{X}(\Omega)$ by hypothesis. Fatou's lemma also shows that $\hat{\varphi}$ is a bounded set in $L_{w}^{N}(\Omega)$. Moreover, since $N>1$, if $\left\{f^{j}\right\}$ is bounded in $L_{w}^{N}(\Omega)$ and $f^{j} \rightarrow f$ a.e.- $w$, then $\left(f^{j}\right)_{E, w} \rightarrow f_{E, w}$ for all $E \in \Sigma$; in fact, in this situation, by using Egorov's theorem, we have $\int_{\Omega} f^{j} \varphi d w \rightarrow \int_{\Omega} f \varphi d w$ for all $\varphi \in L_{w}^{N^{\prime}}(\Omega), 1 / N+1 / N^{\prime}=1$.

Next, while the hypothesis $w\left(E_{l}\right)>0$ in assumption (i) ensures that the averages $f_{E_{l}, w}$ in (1-4) are well-defined, it is not needed since we can discard any pair 
$E_{l}, F_{l}$ with $w\left(E_{l}\right)=0$ without affecting the inequality $w\left(\Omega \backslash \bigcup E_{l}\right)<\epsilon$ or (1-3) and (1-4).

Finally, since $\hat{\mathscr{Y}}$ contains the first component $f$ of any pair $(f, \boldsymbol{g}) \in \mathscr{Y}$, a simple corollary of Theorem 1.1 is that the projection $\pi$ defined in (1-2) is a compact mapping of $\mathscr{S}$ into $L_{w}^{q}(\Omega), 1 \leq q<N$, in the sense that, for every sequence $\left\{\left(f_{k}, \boldsymbol{g}_{k}\right)\right\} \subset \mathscr{Y}$, there is a subsequence $\left\{f_{k_{i}}\right\}$ and a function $f \in L_{w}^{N}(\Omega)$ such that $f_{k_{i}} \rightarrow f$ pointwise a.e.- $w$ in $\Omega$ and in $L_{w}^{q}(\Omega)$ norm for $1 \leq q<N$.

Proof of Theorem 1.1. Let $\mathscr{S}$ satisfy the hypotheses and suppose $\left\{f_{k}\right\}_{k \in \mathbb{N}} \subset \hat{\mathscr{S}}$. For each $f_{k}$, use the definition of $\hat{\mathscr{S}}$ to choose a sequence $\left\{\left(f_{k}^{j}, \boldsymbol{g}_{k}^{j}\right)\right\}_{j} \subset \mathscr{S}$ with $f_{k}^{j} \rightarrow f_{k}$ a.e. $w$ as $j \rightarrow \infty$. Since $\mathscr{S}$ is bounded in $L_{w}^{N}(\Omega) \times \mathscr{L}(\Omega)$, there is $M \in(0, \infty)$ such that

$$
\left\|\left(f_{k}^{j}, \boldsymbol{g}_{k}^{j}\right)\right\|_{L_{w}^{N}(\Omega) \times \mathscr{L}(\Omega)} \leq M
$$

for all $k$ and $j$. Also, as noted above, $\left\{f_{k}\right\}$ is bounded in $L_{w}^{N}(\Omega)$ norm; in fact $\left\|f_{k}\right\|_{L_{w}^{N}(\Omega)} \leq M$ for the same constant $M$ and all $k$.

Since $\left\{f_{k}\right\}$ is bounded in $L_{w}^{N}(\Omega)$, if $1<N<\infty$, it has a weakly convergent subsequence, while if $N=\infty$, it has a subsequence which converges in the weakstar topology. In either case, we relabel the subsequence as $\left\{f_{k}\right\}$ to preserve the index. Fix $\epsilon>0$ and let $\left\{E_{l}, F_{l}\right\}_{l=1}^{J}$ satisfy the hypotheses of the theorem relative to $\epsilon$. Setting $\Omega^{\epsilon}=\bigcup E_{l}$, we have by assumption (i) that

$$
w\left(\Omega \backslash \Omega^{\epsilon}\right)<\epsilon .
$$

Let us show that there is a positive constant $C$ independent of $\epsilon$ such that

$$
\sum_{l}\left\|f_{k}-\left(f_{k}\right)_{E_{l}, w}\right\|_{L_{w}^{p}\left(E_{l}\right)}^{p} \leq C \epsilon^{p} \quad \text { for all } k .
$$

Fix $k$ and let $\Delta$ denote the expression on the left side of (1-7). Since

$$
f_{k}^{j}-\left(f_{k}^{j}\right)_{E_{l}, w} \rightarrow f_{k}-\left(f_{k}\right)_{E_{l}, w}
$$

a.e.- $w$ as $j \rightarrow \infty$, Fatou's lemma gives

$$
\Delta \leq \sum_{l} \liminf _{j \rightarrow \infty}\left\|f_{k}^{j}-\left(f_{k}^{j}\right)_{E_{l}, w}\right\|_{L_{w}^{p}\left(E_{l}\right)}^{p} .
$$

Consequently, by using the Poincaré inequality (1-4) for $\mathscr{S}$ and superadditivity of liminf, we obtain

$$
\Delta \leq \liminf _{j \rightarrow \infty} \sum_{l} \epsilon^{p}\left\|g_{k}^{j} \chi_{F_{l}}\right\|_{\mathscr{X}(\Omega)}^{p}
$$

By (1-3), the sets $F_{l}$ have finite overlaps uniformly in $\epsilon$, with the same overlap constant $C_{1}$ as in property $\left(\mathrm{B}_{p}\right)$ of $\mathscr{L}(\Omega)$. Hence, by applying property $\left(\mathrm{B}_{p}\right)$ to the last expression together with boundedness of $\mathscr{Y}$, we get 


$$
\Delta \leq C_{2} \epsilon^{p} \liminf _{j \rightarrow \infty}\left\|\boldsymbol{g}_{k}^{j}\right\|_{\mathscr{L}(\Omega)}^{p} \leq C_{2} M^{p} \epsilon^{p} .
$$

This proves (1-7) with $C=C_{2} M^{p}$.

Next note that

$$
\int_{\Omega^{\epsilon}}\left|f_{m}-f_{k}\right|^{p} d w \leq \sum_{l} \int_{E_{l}}\left|f_{m}-f_{k}\right|^{p} d w \leq 2^{p-1}(I+I I),
$$

where

$$
I:=\sum_{l} \int_{E_{l}}\left|f_{m}-f_{k}-\left(f_{m}-f_{k}\right)_{E_{l}, w}\right|^{p} d w, \quad I I:=\sum_{l}\left|\left(f_{m}-f_{k}\right)_{E_{l}, w}\right|^{p} w\left(E_{l}\right) .
$$

We estimate $I$ and $I I$ separately. We have

$$
\begin{aligned}
I & \leq 2^{p-1}\left(\sum_{l}\left\|f_{m}-\left(f_{m}\right)_{E_{l}, w}\right\|_{L_{w}^{p}\left(E_{l}\right)}^{p}+\sum_{l}\left\|f_{k}-\left(f_{k}\right)_{E_{l}, w}\right\|_{L_{w}^{p}\left(E_{l}\right)}^{p}\right) \\
& \leq 2^{p-1}\left(C \epsilon^{p}+C \epsilon^{p}\right)=2^{p} C \epsilon^{p},
\end{aligned}
$$

by (1-7). To estimate $I I$, first note that

$$
I I=\sum_{l=1}^{J}\left|\left(f_{m}-f_{k}\right)_{E_{l}, w}\right|^{p} w\left(E_{l}\right)=\sum_{l=1}^{J} \frac{1}{w\left(E_{l}\right)^{p-1}}\left|\int_{\Omega}\left(f_{m}-f_{k}\right) \chi_{E_{l}} d w\right|^{p} .
$$

Since $w(\Omega)<\infty$, each characteristic function $\chi_{E_{l}} \in L_{w}^{N^{\prime}}(\Omega), 1 / N+1 / N^{\prime}=1$ (with $N^{\prime}=1$ if $N=\infty$ ). As $\left\{f_{k}\right\}$ converges weakly in $L_{w}^{N}(\Omega)$ when $1<N<\infty$ or converges in the weak-star sense when $N=\infty$, for $m, k$ sufficiently large depending on $\epsilon$, and for all $1 \leq l \leq J$,

$$
\frac{1}{w\left(E_{l}\right)^{p-1}}\left|\int_{\Omega}\left(f_{m}-f_{k}\right) \chi_{E_{l}} d w\right|^{p} \leq \frac{\epsilon^{p}}{J} .
$$

Thus $I I \leq \epsilon^{p}$ for $m, k$ sufficiently large depending on $\epsilon$. Combining this estimate with (1-8) and (1-9) shows that

$$
\left\|f_{m}-f_{k}\right\|_{L_{w}^{p}\left(\Omega^{\epsilon}\right)}<C \epsilon
$$

for $m, k$ sufficiently large and $C=C\left(M, C_{2}\right)$.

Let us now show that $\left\{f_{k}\right\}$ is a Cauchy sequence in $L_{w}^{1}(\Omega)$. For $m, k$ as in (1-10), Hölder's inequality and the fact that $\left\|f_{k}\right\|_{L_{w}^{N}(\Omega)} \leq M$ for all $k$ yield $\left\|f_{m}-f_{k}\right\|_{L_{w}^{1}(\Omega)}$

$$
\begin{aligned}
& \leq\left\|f_{m}-f_{k}\right\|_{L_{w}^{1}\left(\Omega^{\epsilon}\right)}+\left\|f_{m}-f_{k}\right\|_{L_{w}^{1}\left(\Omega \backslash \Omega^{\epsilon}\right)} \\
& \leq\left\|f_{m}-f_{k}\right\|_{L_{w}^{p}\left(\Omega^{\epsilon}\right)} w\left(\Omega^{\epsilon}\right)^{1 / p^{\prime}}+\left\|f_{m}-f_{k}\right\|_{L_{w}^{N}\left(\Omega \backslash \Omega^{\epsilon}\right)} w\left(\Omega \backslash \Omega^{\epsilon}\right)^{1 / N^{\prime}} \\
& <C \epsilon w\left(\Omega^{\epsilon}\right)^{1 / p^{\prime}}+2 M w\left(\Omega \backslash \Omega^{\epsilon}\right)^{1 / N^{\prime}} \\
& <C \epsilon w(\Omega)^{1 / p^{\prime}}+2 M \epsilon^{1 / N^{\prime}} \quad \text { by }(1-6) .
\end{aligned}
$$


Since $N^{\prime}<\infty$, it follows that $\left\{f_{k}\right\}$ is Cauchy in $L_{w}^{1}(\Omega)$. Hence it has a subsequence (again denoted by $\left\{f_{k}\right\}$ ) that converges in $L_{w}^{1}(\Omega)$ and pointwise a.e.- $w$ in $\Omega$ to a function $f \in L_{w}^{1}(\Omega)$. If $N=\infty,\left\{f_{k}\right\}$ is bounded in $L_{w}^{\infty}(\Omega)$ by hypothesis, so its pointwise limit $f \in L_{w}^{\infty}(\Omega)$. If $N<\infty$, since $\left\{f_{k}\right\}$ is bounded in $L_{w}^{N}(\Omega)$, Fatou's Lemma implies that $f \in L_{w}^{N}(\Omega)$. This completes the proof in case $q=1$.

For general $q$, we use the same subsequence $\left\{f_{k}\right\}$ as above. Thus we only need to show that $\left\{f_{k}\right\}$ converges in $L_{w}^{q}(\Omega)$ for $1<q<N$. We use Hölder's inequality. Given $q \in(1, N)$, choose $\lambda \in(0,1)$, namely, $\lambda=(1 / q-1 / N) /(1-1 / N)$. Hence $\lambda=1 / q$ if $N=\infty$. Then

$$
\left\|f_{m}-f_{k}\right\|_{L_{w}^{q}(\Omega)} \leq\left\|f_{m}-f_{k}\right\|_{L_{w}^{1}(\Omega)}^{\lambda}\left\|f_{m}-f_{k}\right\|_{L_{w}^{N}(\Omega)}^{1-\lambda} .
$$

As before, $\left\|f_{k}\right\|_{L_{w}^{N}(\Omega)} \leq M$, and therefore

$$
\left\|f_{m}-f_{k}\right\|_{L_{w}^{N}(\Omega)}^{1-\lambda} \leq(2 M)^{1-\lambda} .
$$

Hence, by (1-11), $\left\{f_{k}\right\}$ is Cauchy in $L_{w}^{q}(\Omega)$ as it is Cauchy in $L_{w}^{1}(\Omega)$. This completes the proof of Theorem 1.1.

A compact embedding result is also proved in [Franchi et al. 1997, Theorem 3.4] by using Poincaré type estimates. However, Theorem 1.1 applies to situations not considered in [Franchi et al. 1997] since it is not restricted to the context of Lipschitz vector fields in $\mathbb{R}^{n}$. Other abstract compact embedding results can be found in [Hajłasz and Koskela 1998, Theorem 4; Hajłasz and Koskela 2000, Theorem 8.1], including a version [Hajłasz and Koskela 1998, Theorem 5] for weighted Sobolev spaces with nonzero continuous weights, and a version [Hajłasz and Koskela 2000] for metric spaces with a single doubling measure. The proof in [Hajłasz and Koskela 1998] assumes prior knowledge of the classical Rellich-Kondrachov compactness theorem (see, for example, [Gilbarg and Trudinger 1997, Theorem 7.22(i)] and below).

By making minor changes in the proof of Theorem 1.1, we can obtain a sufficient condition for a bounded set in $L_{w}^{N}(\Omega)$ to be precompact in $L_{w}^{q}(\Omega), 1 \leq q<N$, without mentioning the sets $\left\{F_{l}\right\}$, the space $\mathscr{L}(\Omega)$, properties $(\mathrm{A})$ and $\left(\mathrm{B}_{p}\right)$, or conditions (1-3) and (1-4). We state this result in the next theorem. An application is given in Section 4.

Theorem 1.2. Let $w$ be a finite measure on a $\sigma$-algebra $\Sigma$ of subsets of a set $\Omega$, with $\Omega \in \Sigma$. Let $1 \leq p<\infty, 1<N \leq \infty$, and $\mathscr{P}$ be a bounded subset of $L_{w}^{N}(\Omega)$. Suppose there is a positive constant $C$ such that, for every $\epsilon>0$, there are a finite number of sets $E_{l} \in \Sigma$ with

(i) $w\left(\Omega \backslash \bigcup_{l} E_{l}\right)<\epsilon$ and $w\left(E_{l}\right)>0$; 
(ii) for every $f \in \mathscr{P}$,

$$
\sum_{l}\left\|f-f_{E_{l}, w}\right\|_{L_{w}^{p}\left(E_{l}\right)}^{p} \leq C \epsilon^{p}
$$

Let

$$
\widehat{\mathscr{P}}=\left\{f \in L_{w}^{N}(\Omega): \text { there exists }\left\{f^{j}\right\} \subset \mathscr{P} \text { with } f^{j} \rightarrow f \text { a.e. }-w\right\} .
$$

Then, for every sequence $\left\{f_{k}\right\} \subset \widehat{\mathscr{P}}$, there is a single subsequence $\left\{f_{k_{i}}\right\}$ and a function $f \in L_{w}^{N}(\Omega)$ such that $f_{k_{i}} \rightarrow f$ pointwise a.e.- $w$ in $\Omega$ and in $L_{w}^{q}(\Omega)$ norm for $1 \leq q<N$.

Remark 1.3. (1) Given $\epsilon>0$, let $\left\{E_{l}\right\}$ satisfy hypothesis (i) of Theorem 1.2. Hypothesis (ii) of Theorem 1.2 is clearly true for $\left\{E_{l}\right\}$ if, for every $f \in \mathscr{P}$, there are nonnegative constants $\left\{a_{l}\right\}$ such that

$$
\left\|f-f_{E_{l}, w}\right\|_{L_{w}^{p}\left(E_{l}\right)} \leq \epsilon a_{l}
$$

and

$$
\sum a_{l}^{p} \leq C
$$

with $C$ independent of $f, \epsilon$. The constants $\left\{a_{l}\right\}$ may vary with $f$ and $\epsilon$.

(2) Theorem 1.1 is a corollary of Theorem 1.2. To see why, suppose that the hypothesis of Theorem 1.1 holds. Define

$$
\mathscr{P}=\pi(\mathscr{S})=\{f:(f, \boldsymbol{g}) \in \mathscr{Y}\} .
$$

Let $\epsilon>0$ and choose $\left\{\left(E_{l}, F_{l}\right)\right\}$ as in Theorem 1.1. Given $f \in \mathscr{P}$, choose any $\boldsymbol{g}$ such that $(f, \boldsymbol{g}) \in \mathscr{Y}$ and set $a_{l}=\left\|\boldsymbol{g} \chi_{F_{l}}\right\|_{\mathscr{X}(\Omega)}$ for all $l$. Then (1-4), (1-3), and property $\left(\mathrm{B}_{p}\right)$ of $\mathscr{X}(\Omega)$ imply (1-13) and (1-14). The preceding remark shows that the hypothesis of Theorem 1.2 holds. The conclusion of Theorem 1.1 now follows from Theorem 1.2.

Proof of Theorem 1.2. Theorem 1.2 can be proved by checking through the proof of Theorem 1.1. In fact, the nature of hypothesis (1-12) allows simplification of the proof. First recall that if $f^{j} \rightarrow f$ a.e.- $w$ and $\left\{f^{j}\right\}$ is bounded in $L_{w}^{N}(\Omega)$, $\left(f^{j}\right)_{E, w} \rightarrow f_{E, w}$ for every $E \in \Sigma$. Therefore, by the definition of $\widehat{\mathscr{P}}$ and Fatou's lemma, the truth of (1-12) for all $f \in \mathscr{P}$ implies its truth for all $f \in \widehat{\mathscr{P}}$. Given a sequence $\left\{f_{k}\right\}$ in $\widehat{\mathscr{P}}$, we follow the proof of Theorem 1.1, but we no longer need to introduce the $\left\{f_{k}^{j}\right\}$ or prove (1-7) since (1-7) now follows from the fact that (1-12) holds for $\widehat{\mathscr{P}}$. Further details are left to the reader.

We close this section by listing an alternate version of Theorem 1.1 that we use in Section 3D when we consider local results. 
Theorem 1.4. Let $w$ be a measure (not necessarily finite) on a $\sigma$-algebra $\Sigma$ of subsets of a set $\Omega$, with $\Omega \in \Sigma$. Let $1 \leq p<\infty, 1<N \leq \infty$, $\mathscr{L}(\Omega)$ be a normed linear space satisfying properties $(\mathrm{A})$ and $\left(\mathrm{B}_{p}\right)$ relative to a set $\Sigma_{0} \subset \Sigma$, and let $\mathscr{Y}$ be a collection of pairs $(f, \boldsymbol{g})$ such that $f$ is $\Sigma$-measurable and $\boldsymbol{g} \in \mathscr{L}(\Omega)$.

Suppose that $\mathscr{Y}$ satisfies the following conditions relative to a fixed set $\Omega^{\prime} \in \Sigma$ (in particular $\Omega^{\prime} \subset \Omega$ ): for each $\epsilon=\epsilon_{j}=1 / j$ with $j \in \mathbb{N}$, there are a finite number of pairs $\left\{E_{l}^{\epsilon}, F_{l}^{\epsilon}\right\}_{l}$ with $E_{l}^{\epsilon} \in \Sigma$ and $F_{l}^{\epsilon} \in \Sigma_{0}$ satisfying these conditions:

(i) $w\left(\Omega^{\prime} \backslash \bigcup_{l} E_{l}^{\epsilon}\right)=0$ and $0<w\left(E_{l}^{\epsilon}\right)<\infty$.

(ii) $\left\{F_{l}^{\epsilon}\right\}_{l}$ has bounded overlaps independent of $\epsilon$ with the same overlap constant as in $\left(\mathrm{B}_{p}\right)$, that is,

$$
\sum_{l} \chi_{F_{l}^{\epsilon}}(x) \leq C_{1}, \quad x \in \Omega
$$

for $C_{1}$ as in $\left(\mathrm{B}_{p}\right)$.

(iii) For every $(f, \boldsymbol{g}) \in \mathscr{Y}$, the local Poincaré-type inequality

$$
\left\|f-f_{E_{l}^{\epsilon}, w}\right\|_{L_{w}^{p}\left(E_{l}^{\epsilon}\right)} \leq \epsilon\left\|\boldsymbol{g} \chi_{F_{l}^{\epsilon}}\right\|_{\mathscr{X}(\Omega)}
$$

holds for each $\left(E_{l}^{\epsilon}, F_{l}^{\epsilon}\right)$.

Then, for every sequence $\left\{\left(f_{k}, \boldsymbol{g}_{k}\right)\right\}$ in $\mathscr{S}$ with

$$
\sup _{k}\left[\left\|f_{k}\right\|_{L_{w}^{N}\left(\cup_{l, j} E_{l}^{1 / j}\right)}+\left\|\boldsymbol{g}_{k}\right\|_{\mathscr{X}(\Omega)}\right]<\infty,
$$

there is a subsequence $\left\{f_{k_{i}}\right\}$ of $\left\{f_{k}\right\}$ and a function $f \in L_{w}^{N}\left(\Omega^{\prime}\right)$ such that $f_{k_{i}} \rightarrow f$ pointwise a.e. $-w$ in $\Omega^{\prime}$ and in $L_{w}^{q}\left(\Omega^{\prime}\right)$ norm for $1 \leq q \leq p$. If $p<N$, then also $f_{k_{i}} \rightarrow f$ in $L_{w}^{q}\left(\Omega^{\prime}\right)$ norm for $1 \leq q<N$.

The principal difference between the assumptions in Theorems 1.1 and 1.4 occurs in hypothesis (i). When we apply Theorem 1.4 in Section 3D, the sets $\left\{E_{l}^{\epsilon}\right\}$ will satisfy $\Omega^{\prime} \subset \bigcup_{l} E_{l}^{\epsilon}$ for each $\epsilon$, and consequently the condition in hypothesis (i) that $w\left(\Omega^{\prime} \backslash \bigcup_{l} E_{l}^{\epsilon}\right)=0$ for each $\epsilon$ will automatically be true. Unlike Theorem 1.1, the value of $q$ in Theorem 1.4 is always allowed to equal $p$. Although $w(\Omega)$ is not assumed to be finite in Theorem 1.4,w $\left(\Omega^{\prime}\right)<\infty$ is true due to hypothesis (i) and the fact that the number of $E_{l}^{\epsilon}$ is finite for each $\epsilon$. As in Theorem 1.1, the hypothesis $w\left(E_{l}^{\epsilon}\right)>0$ is dispensable.

Proof of Theorem 1.4. The proof is like that of Theorem 1.1, with minor changes and some simplifications. We work directly with the pairs $\left(f_{k}, \boldsymbol{g}_{k}\right)$ without considering approximations $\left(f_{k}^{j}, \boldsymbol{g}_{k}^{j}\right)$. Due to the form of assumption (i) in Theorem 1.4, neither the set $\Omega^{\epsilon}$ nor estimate (1-6) is now needed. Since $w\left(\Omega^{\prime} \backslash \bigcup_{l} E_{l}^{\epsilon}\right)=0$ for each $\epsilon=1 / j$, we can replace $\Omega^{\epsilon}$ by $\Omega^{\prime}$ in the proof, obtaining the estimate

$$
\left\|f_{m}-f_{k}\right\|_{L_{w}^{p}\left(\Omega^{\prime}\right)}<C \epsilon
$$


as an analogue of (1-10). In deriving (1-16), the weak and weak-star arguments are guaranteed since, by (1-15),

$$
\sup _{k}\left\|f_{k}\right\|_{L_{w}^{N}}\left(\bigcup_{l, j} E_{l}^{1 / j}\right)<\infty
$$

The main change in the proof comes by observing that the entire argument formerly used to show that $\left\{f_{k}\right\}$ is Cauchy in $L_{w}^{1}(\Omega)$ is no longer needed. In fact, (1-16) proves that $\left\{f_{k}\right\}$ is Cauchy in $L_{w}^{p}\left(\Omega^{\prime}\right)$, and therefore it is also Cauchy in $L_{w}^{q}\left(\Omega^{\prime}\right)$ if $1 \leq q \leq p$ since $w\left(\Omega^{\prime}\right)<\infty$. The first conclusion in Theorem 1.4 then follows. To prove the second one, assuming that $p, q<N$, we use an analogue of (1-11) with $\Omega^{\prime}$ in place of $\Omega$ and the same choice of $\lambda$, namely,

$$
\left\|f_{m}-f_{k}\right\|_{L_{w}^{q}\left(\Omega^{\prime}\right)} \leq\left\|f_{m}-f_{k}\right\|_{L_{w}^{1}\left(\Omega^{\prime}\right)}^{\lambda}\left\|f_{m}-f_{k}\right\|_{L_{w}^{N}\left(\Omega^{\prime}\right)}^{1-\lambda}
$$

The desired conclusion then follows as before since we have already shown that the first factor on the right side tends to 0 .

\section{Applications in the nondegenerate case}

Roughly speaking, a consequence of Theorem 1.1 is that a set of functions which is bounded in $L_{w}^{N}(\Omega)$ is precompact in $L_{w}^{q}(\Omega)$ for $1 \leq q<N$ if the gradients of the functions are bounded in an appropriate norm and a local Poincaré inequality holds for them. The requirement of boundedness in $L_{w}^{N}(\Omega)$ will be fulfilled if, for example, the functions satisfy a global Poincaré or Sobolev estimate with exponent $N$ on the left side. In order to illustrate this principle more precisely, we first consider the classical gradient operator and functions on $\mathbb{R}^{n}$ with the standard Euclidean metric. We include a simple way to see that the Rellich-Kondrachov compactness theorem follows from our results. Our derivation of this fact is different from those in [Adams and Fournier 2003; Gilbarg and Trudinger 1997]; in particular, it avoids using the Arzelá-Ascoli theorem and regularization of functions by convolution. We also list compactness results for the special class of $s$-John domains in $\mathbb{R}^{n}$. Hajlasz and Koskela [1998] mention that such results follow from their development without giving specific statements. See also [Hajłasz and Koskela 2000, Theorem 8.1]. We list results for degenerate quadratic forms and vector fields in Section 3.

We begin by proving a compact embedding result for some Sobolev spaces involving two measures. Let $w$ be a measure on the Borel subsets of a fixed open set $\Omega \subset \mathbb{R}^{n}$, and let $\mu$ be a measure on the $\sigma$-algebra of Lebesgue measurable subsets of $\Omega$. We also assume that $\mu$ is absolutely continuous with respect to Lebesgue measure. If $1 \leq p<\infty$, let $E_{\mu}^{p}(\Omega)$ denote the class of locally Lebesgue integrable functions on $\Omega$ with distributional derivatives in $L_{\mu}^{p}(\Omega)$. If $1 \leq N \leq \infty$, we say that a set $Y \subset L_{w}^{N}(\Omega) \cap E_{\mu}^{p}(\Omega)$ (intersection of function spaces instead of normed 
spaces of equivalence classes) is bounded in $L_{w}^{N}(\Omega) \cap E_{\mu}^{p}(\Omega)$ if

$$
\sup _{f \in Y}\left\{\|f\|_{L_{w}^{N}(\Omega)}+\|\nabla f\|_{L_{\mu}^{p}(\Omega)}\right\}<\infty .
$$

We use $D$ to denote a generic open Euclidean ball. The radius and center of $D$ will be denoted $r(D)$ and $x_{D}$, and if $C$ is a positive constant, $C D$ will denote the ball concentric with $D$ whose radius is $\operatorname{Cr}(D)$.

Theorem 2.1. Let $\widetilde{\Omega} \subset \Omega$ be open sets in $\mathbb{R}^{n}$. Let $w$ be a Borel measure on $\Omega$ with $w(\widetilde{\Omega})=w(\Omega)<\infty$ and $\mu$ be a measure on the Lebesgue measurable sets in $\Omega$ which is absolutely continuous with respect to Lebesgue measure. Let $1 \leq p<\infty$, $1<N \leq \infty$, and $\mathscr{Y} \subset L_{w}^{N}(\Omega) \cap E_{\mu}^{p}(\Omega)$, and suppose that, for all $\epsilon>0$, there exists $\delta_{\epsilon}>0$ such that

$$
\left\|f-f_{D, w}\right\|_{L_{w}^{p}(D)} \leq \epsilon\|\nabla f\|_{L_{\mu}^{p}(D)} \quad \text { for all } f \in \mathscr{S}
$$

and all Euclidean balls $D$ with $r(D)<\delta_{\epsilon}$ and $2 D \subset \tilde{\Omega}$. Then, for any sequence $\left\{f_{k}\right\} \subset \mathscr{Y}$ that is bounded in $L_{w}^{N}(\Omega) \cap E_{\mu}^{p}(\Omega)$, there is a subsequence $\left\{f_{k_{i}}\right\}$ and a function $f \in L_{w}^{N}(\Omega)$ such that $\left\{f_{k_{i}}\right\} \rightarrow f$ pointwise a.e. $-w$ in $\Omega$ and in $L_{w}^{q}(\Omega)$ norm for $1 \leq q<N$.

Before proving Theorem 2.1, we give typical examples of $\widetilde{\Omega}$ and $w$ with $w(\widetilde{\Omega})=$ $w(\Omega)<\infty$. For any two nonempty sets $E_{1}, E_{2} \subset \mathbb{R}^{n}$, let

$$
\rho\left(E_{1}, E_{2}\right)=\inf \left\{|x-y|: x \in E_{1}, y \in E_{2}\right\}
$$

denote the Euclidean distance between $E_{1}$ and $E_{2}$. If $x \in \mathbb{R}^{n}$ and $E$ is a nonempty set, we write $\rho(x, E)$ instead of $\rho(\{x\}, E)$. Let $\widetilde{\Omega}$ be an open subset of $\Omega$. If $\Omega$ is bounded and $\Omega \backslash \widetilde{\Omega}$ has Lebesgue measure 0 , the measure $w$ on $\Omega$ defined by $d w=\rho\left(x, \mathbb{R}^{n} \backslash \tilde{\Omega}\right)^{\alpha} d x$ clearly has the desired properties if $\alpha \geq 0$. The range of $\alpha$ can be increased to $\alpha>-1$ if $\Omega$ is a Lipschitz domain and $\Omega \backslash \widetilde{\Omega}$ is a finite set. Indeed, if $\partial \Omega$ is described in local coordinates $x=\left(x_{1}, \ldots, x_{n}\right)$ by $x_{n}=F\left(x_{1}, \ldots, x_{n-1}\right)$ with $F$ Lipschitz, the distance from $x$ to $\partial \Omega$ is equivalent to $\left|x_{n}-F\left(x_{1}, \ldots, x_{n-1}\right)\right|$, and, consequently, the restriction $\alpha>-1$ guarantees that $w$ is finite near $\partial \Omega$ by using Fubini's theorem; see also [Chua 1995, Remark 3.4(b)]. If $\Omega$ is bounded and $\Omega \backslash \widetilde{\Omega}$ is finite, but with no restriction on $\partial \Omega$, the range can clearly be further increased to $\alpha>-n$ for the measure $\rho(x, \Omega \backslash \widetilde{\Omega})^{\alpha} d x$. Also note that any $w$ without point masses satisfies $w(\widetilde{\Omega})=w(\Omega)$ if $\widetilde{\Omega}$ is obtained by deleting a countable subset of $\Omega$.

Proof of Theorem 2.1. We verify the hypotheses of Theorem 1.1. Let

$$
\mathscr{X}(\Omega)=\left\{\boldsymbol{g}=\left(g_{1}, \ldots, g_{n}\right):|\boldsymbol{g}|=\left(\sum_{i=1}^{n} g_{i}^{2}\right)^{1 / 2} \in L_{\mu}^{p}(\Omega)\right\}
$$


and $\|\boldsymbol{g}\|_{\mathscr{L}(\Omega)}=\|\boldsymbol{g}\|_{L_{\mu}^{p}(\Omega)}$. Then

$$
\|\nabla f\|_{\mathscr{L}(\Omega)}=\|\nabla f\|_{L_{\mu}^{p}(\Omega)} \quad \text { if } f \in E_{\mu}^{p}(\Omega) .
$$

If $f \in E_{\mu}^{p}(\Omega)$, we may identify $f$ with the pair $(f, \nabla f)$ since the distributional gradient $\nabla f$ is uniquely determined by $f$ up to a set of Lebesgue measure zero. Then $L_{w}^{N}(\Omega) \cap E_{\mu}^{p}(\Omega)$ can be viewed as a subset of $L_{w}^{N}(\Omega) \times \mathscr{X}(\Omega)$. In Theorem 1.1, choose $\mathscr{S}$ to be the particular sequence $\left\{f_{k}\right\} \subset \mathscr{Y}$ in the hypothesis of Theorem 2.1, $\Sigma$ to be the Lebesgue measurable subsets of $\Omega$, and $\Sigma_{0}$ to be the collection of balls $D \subset \Omega$. Then hypotheses (A) and $\left(\mathrm{B}_{p}\right)$ are valid with $C_{2}=C_{1}$ for any $C_{1}$. Given $\epsilon>0$, since $w(\widetilde{\Omega})=w(\Omega)<\infty$, there is a compact set $K \subset \widetilde{\Omega}$ with $w(\Omega \backslash K)<\epsilon$. Let $0<\delta_{\epsilon}^{\prime}<\rho\left(K, \mathbb{R}^{n} \backslash \widetilde{\Omega}\right)$ (where $\rho\left(K, \mathbb{R}^{n} \backslash \widetilde{\Omega}\right)$ is interpreted as $\infty$ if $\widetilde{\Omega}=\mathbb{R}^{n}$ ). Let $\delta_{\epsilon}$ be as in (2-1), and fix $r_{\epsilon}$ with $0<r_{\epsilon}<\min \left\{\delta_{\epsilon}, \delta_{\epsilon}^{\prime}\right\}$. By considering the triples of balls in a maximal collection of pairwise disjoint balls of radius $r_{\epsilon} / 6$ centered in $K$, we obtain a collection $\left\{E_{l}^{\epsilon}\right\}_{l}$ of balls of radius $r_{\epsilon} / 2$ which satisfy $2 E_{l}^{\epsilon} \subset \widetilde{\Omega}$, have bounded overlaps with overlap constant independent of $\epsilon$, and whose union covers $K$. Since $K$ is compact, we may assume the collection is finite. Also,

$$
w\left(\Omega \backslash \bigcup_{l} E_{l}^{\epsilon}\right) \leq w(\Omega \backslash K)<\epsilon,
$$

and (1-4) holds with $F_{l}=E_{l}=E_{l}^{\epsilon}$ by (2-1). Theorem 2.1 now follows from Theorem 1.1 applied to $\Omega$.

In particular, we obtain the following result when $w=\mu$ is a Muckenhoupt $A_{p}\left(\mathbb{R}^{n}\right)$ weight, that is, when $d \mu=d w=\eta d x$, where $\eta(x)$ satisfies

$$
\left(\frac{1}{|D|} \int_{D} \eta d x\right)\left(\frac{1}{|D|} \int_{D} \eta^{-1 /(p-1)} d x\right)^{p-1} \leq C
$$

if $1<p<\infty$, and satisfies $|D|^{-1} \int_{D} \eta d x \leq C \operatorname{essinf}_{D} w$ if $p=1$, for all Euclidean balls $D$, with $C$ independent of $D$. As is well known, such a weight also satisfies the classical doubling condition

$$
w\left(D_{r}(x)\right) \leq C\left(\frac{r}{r^{\prime}}\right)^{\theta} w\left(D_{r^{\prime}}(x)\right), \quad 0<r^{\prime}<r<\infty,
$$

with $\theta=n p-\epsilon$ for some $\epsilon>0$ if $p>1$, and with $\theta=n$ if $p=1$, where $C$ and $\epsilon$ are independent of $r, r^{\prime}, x$.

We denote by $W^{1, p, w}(\Omega)$ the weighted Sobolev space defined as all functions in $L_{w}^{p}(\Omega)$ whose distributional gradient is in $L_{w}^{p}(\Omega)$. Therefore $W^{1, p, w}(\Omega)=$ $L_{w}^{p}(\Omega) \cap E_{w}^{p}(\Omega)$. If $w(\Omega)<\infty$, it follows that $L_{w}^{N}(\Omega) \cap E_{w}^{p}(\Omega) \subset W^{1, p, w}(\Omega)$ when $N \geq p$, and that the opposite containment holds when $N \leq p$.

Theorem 2.2. Let $1 \leq p<\infty, w \in A_{p}\left(\mathbb{R}^{n}\right)$, and $\Omega$ be an open set in $\mathbb{R}^{n}$ with $w(\Omega)<\infty$. If $1<N \leq \infty$, then any bounded subset of $L_{w}^{N}(\Omega) \cap E_{w}^{p}(\Omega)$ is 
precompact in $L_{w}^{q}(\Omega)$ if $1 \leq q<N$. Consequently, if $N>p$ and $\mathscr{Y}$ is a subset of $W^{1, p, w}(\Omega)$ with

$$
\|f\|_{L_{w}^{N}(\Omega)} \leq C\left(\|f\|_{L_{w}^{p}(\Omega)}+\|\nabla f\|_{L_{w}^{p}(\Omega)}\right) \quad \text { for all } f \in \mathscr{Y},
$$

then any set in $\mathscr{Y}$ that is bounded in $W^{1, p, w}(\Omega)$ is precompact in $L_{w}^{q}(\Omega)$ for $1 \leq q<N$.

If $\Omega$ is a John domain, then there exists $N>p(N$ can be $\theta p /(\theta-p)$ for some $\theta>p$ as described after (2-3)) such that $W^{1, p, w}(\Omega)$ is compactly embedded in $L_{w}^{q}(\Omega)$ for $1 \leq q<N$. In particular, the embedding of $W^{1, p, w}(\Omega)$ into $L_{w}^{p}(\Omega)$ is compact when $w \in A_{p}\left(\mathbb{R}^{n}\right)$ and $\Omega$ is a John domain.

Remark 2.3. When $w=1$ and $p<n$, the choices $N=n p /(n-p)$ and $\mathscr{Y}=$ $W_{0}^{1, p}(\Omega)$ - the closure in $W^{1, p}(\Omega)$ of the class of Lipschitz functions with compact support in $\Omega$ - guarantee (2-4) by the classical Sobolev inequality for functions in $W_{0}^{1, p}(\Omega)$ (see, for example, [Gilbarg and Trudinger 1997, Theorem 7.10]). Consequently, the classical Rellich-Kondrachov theorem giving the compact embedding of $W_{0}^{1, p}(\Omega)$ in $L^{q}(\Omega)$ for $1 \leq q<n p /(n-p)$ follows as a special case of the first part of Theorem 2.2.

Proof. We apply Theorem 2.1 with $w=\mu$. Fix $p$ and $w$ with $1 \leq p<\infty$ and $w \in A_{p}\left(\mathbb{R}^{n}\right)$. By [Fabes et al. 1982], there is a constant $C$ such that the weighted Poincaré inequality

$$
\left\|f-f_{D, w}\right\|_{L_{w}^{p}(D)} \leq C r(D)\|\nabla f\|_{L_{w}^{p}(D)}, \quad f \in C^{\infty}(\Omega),
$$

holds for all Euclidean balls $D \subset \Omega$. Then since $C^{\infty}(\Omega)$ is dense in $L_{w}^{N}(\Omega) \cap$ $E_{w}^{p}(\Omega)$ if $1 \leq N<\infty$ (see, for example, [Turesson 2000]), by fixing any $\epsilon>0$ we obtain from Fatou's lemma that, for all balls $D \subset \Omega$ with $\operatorname{Cr}(D) \leq \epsilon$,

$$
\left\|f-f_{D, w}\right\|_{L_{w}^{p}(D)} \leq \epsilon\|\nabla f\|_{L_{w}^{p}(D)} \text { if } f \in L_{w}^{N}(\Omega) \cap E_{w}^{p}(\Omega) .
$$

The same holds when $N=\infty$ since $L_{w}^{\infty}(\Omega)=L^{\infty}(\Omega) \subset L_{w}^{p}(\Omega)$ due to the assumptions $w \in A_{p}\left(\mathbb{R}^{n}\right)$ and $w(\Omega)<\infty$. With $1<N \leq \infty$, the first statement of the theorem now follows from Theorem 2.1, and the second statement is a corollary of the first one.

Next, let $\Omega$ be a John domain. Choose $\theta>p$ such that $w$ satisfies (2-3) and define $N=\theta p /(\theta-p)$. Then $N>p$ and, by [Chua and Wheeden 2008, Theorem 1.8(b) or Theorem 4.1],

$$
\left\|f-f_{\Omega, w}\right\|_{L_{w}^{N}(\Omega)} \leq C\|\nabla f\|_{L_{w}^{p}(\Omega)}, \quad \forall f \in C^{\infty}(\Omega) .
$$

Again, the inequality remains true for functions in $W^{1, p, w}(\Omega)$ by density and Fatou's lemma. It is now clear that (2-4) holds, and the last part of the theorem follows. 
Our next example involves domains in $\mathbb{R}^{n}$ which are more restricted. For special $\Omega$, there are values $N>1$ such that

$$
\|f\|_{L^{N}(\Omega)} \leq C\left(\|f\|_{L^{1}(\Omega)}+\|\nabla f\|_{L^{p}(\Omega)}\right)
$$

for all $f \in L^{1}(\Omega) \cap E^{p}(\Omega)$. Note that if $\Omega$ has finite Lebesgue measure, then $W^{1, p}(\Omega) \subset L^{1}(\Omega) \cap E^{p}(\Omega)$. As we will explain, (2-5) is true for some $N>1$ if $\Omega$ is an $s$-John domain in $\mathbb{R}^{n}$ and $1 \leq s<1+p /(n-1)$. Recall that, for $1 \leq s<\infty$, a bounded domain $\Omega \subset \mathbb{R}^{n}$ is called an $s$-John domain with central point $x^{\prime} \in \Omega$ if for some constant $c>0$ and all $x \in \Omega$ with $x \neq x^{\prime}$, there is a curve $\Gamma:[0, l] \rightarrow \Omega$ such that $\Gamma(0)=x, \Gamma(l)=x^{\prime}$,

$$
\begin{array}{ccrl}
\left|\Gamma\left(t_{1}\right)-\Gamma\left(t_{2}\right)\right| & \leq t_{2}-t_{1} & & \text { for all }\left[t_{1}, t_{2}\right] \subset[0, l], \\
\rho\left(\Gamma(t), \Omega^{c}\right) & \geq c t^{s} & & \text { for all } t \in[0, l] .
\end{array}
$$

The terms 1-John domain and John domain are the same. When $\Omega$ is an $s$-John domain for some $s \in[1,1+p /(n-1))$, it is shown in [Kilpeläinen and Malý 2000; Chua and Wheeden 2008; 2011] that (2-5) holds for all finite $N$ with

$$
\frac{1}{N} \geq \frac{s(n-1)-p+1}{n p}
$$

and for all $f \in W^{1, p}(\Omega)$ without any support restrictions. Note that the right side of (2-6) is strictly less than $1 / p$ for such $s$, and consequently there are values $N>p$ which satisfy (2-6). For $N$ as in (2-6), the global estimate

$$
\left\|f-f_{\Omega}\right\|_{L^{N}(\Omega)} \leq C\|\nabla f\|_{L^{p}(\Omega)}, \quad f_{\Omega}=\int_{\Omega} f(x) d x /|\Omega|,
$$

is shown to hold if $f \in \operatorname{Lip}_{\text {loc }}(\Omega)$ [Chua and Wheeden 2011], and then follows for all $f \in L^{1}(\Omega) \cap E^{p}(\Omega)$; see the proof of Theorem 2.4 for related comments. Inequality (2-5) is clearly a consequence of (2-7).

More generally, weighted versions of (2-7) hold for $s$-John domains and lead to weighted compactness results, as we now show. Let $1 \leq p<\infty$, and, for real $\alpha$ and $\rho\left(x, \Omega^{c}\right)$ as in (2-2), let $L_{\rho^{\alpha} d x}^{p}(\Omega)$ be the class of Lebesgue measurable $f$ on $\Omega$ with

$$
\|f\|_{L_{\rho^{\alpha} d x}^{p}(\Omega)}=\left(\int_{\Omega}|f(x)|^{p} \rho\left(x, \Omega^{c}\right)^{\alpha} d x\right)^{1 / p}<\infty .
$$

Theorem 2.4. Suppose that $1 \leq s<\infty$ and $\Omega$ is an $s$-John domain in $\mathbb{R}^{n}$. Let $p, a, b$ satisfy $1 \leq p<\infty, a \geq 0, b \in \mathbb{R}$, and $b-a<p$.

(i) If

$$
n+a>s(n-1+b)-p+1,
$$


then, for any $1 \leq q<\infty$ such that

$$
\frac{1}{q}>\max \left\{\frac{1}{p}-\frac{1}{n}, \frac{s(n-1+b)-p+1}{(n+a) p}\right\},
$$

$L_{\rho^{a} d x}^{1}(\Omega) \cap E_{\rho^{b} d x}^{p}(\Omega)$ is compactly embedded in $L_{\rho^{a} d x}^{q}(\Omega)$.

(ii) If $p>1$ and

$$
n+a p>s(n-1+b)-p+1 \geq n+a,
$$

then, for any $1 \leq q<\infty$ such that

$$
\frac{a}{q}>\max \left\{\frac{b}{p}-1, \frac{s(n-1+b)-p-n+1}{p}\right\},
$$

$L_{\rho^{a} d x}^{1}(\Omega) \cap E_{\rho^{b} d x}^{p}(\Omega)$ is compactly embedded in $L_{\rho^{a} d x}^{q}(\Omega)$.

Remark 2.5. (1) If $a=b=0,(2-8)$ is the same as $s<1+p /(n-1)$. If $a=0$, (2-10) never holds.

(2) The requirement that $b-a<p$ follows from (2-8) and (2-9) by considering the cases $n-1+b \geq 0$ and $n-1+b<0$ separately. Hence $b-a<p$ automatically holds in Theorem 2.4(i), but it is an assumption in (ii). Also, (2-10) and (2-11) imply that $q<p$, and consequently that $p>1$.

(3) Conditions (2-8) and (2-9) imply there exists $N \in(p, \infty)$ with

$$
\frac{1}{q}>\frac{1}{N}>\max \left\{\frac{1}{p}-\frac{1}{n}, \frac{s(n-1+b)-p+1}{(n+a) p}\right\} .
$$

Conversely, (2-8) holds if there exists $N \in(p, \infty)$ such that (2-12) holds.

(4) Assumption (2-11) ensures that there exists $N \in(q, \infty)$ such that (2-11) holds with $q$ replaced by $N$.

Proof of Theorem 2.4. This result is also a consequence of Theorem 2.1, but we deduce it from Theorem 1.1 by using arguments like those in the proofs of Theorems 2.1 and 2.2. Fix $a, b, p, q$ as in the hypothesis and denote $\rho(x)=\rho\left(x, \Omega^{c}\right)$. Choose $w=\rho^{a} d x$ and note that $w(\Omega)<\infty$ since $a \geq 0$ and $\Omega$ is now bounded. Define

$$
\mathscr{L}(\Omega)=\left\{\boldsymbol{g}=\left(g_{1}, \ldots, g_{n}\right):|\boldsymbol{g}| \in L_{\rho^{b} d x}^{p}(\Omega)\right\}
$$

and $\|\boldsymbol{g}\|_{\mathscr{X}(\Omega)}=\|\boldsymbol{g}\|_{L_{\rho^{b} d x}^{p}(\Omega)}$. Fix $\epsilon>0$ and choose a compact set $K \subset \Omega$ with

$$
|\Omega \backslash K|_{\rho^{a} d x}:=\int_{\Omega \backslash K} \rho^{a} d x<\epsilon .
$$

Also choose $\delta_{\epsilon}^{\prime}$ with $0<\delta_{\epsilon}^{\prime}<\rho\left(K, \Omega^{c}\right)$, where $\rho\left(K, \Omega^{c}\right)$ is the Euclidean distance between $K$ and $\Omega^{c}$. 
If $D$ is a Euclidean ball with center $x_{D} \in K$ and $r(D)<\frac{1}{2} \delta_{\epsilon}^{\prime}$, then $2 D \subset \Omega$ and $\rho(x)$ is essentially constant on $D$; in fact, for such $D$,

$$
\frac{1}{2} \rho\left(x_{D}\right) \leq \rho(x) \leq \frac{3}{2} \rho\left(x_{D}\right), \quad x \in D .
$$

We claim that, for such $D$, the simple unweighted Poincaré estimate

$$
\left\|f-f_{D}\right\|_{L^{p}(D)} \leq \operatorname{Cr}(D)\|\nabla f\|_{L^{p}(D)}, \quad f \in \operatorname{Lip}_{\mathrm{loc}}(\Omega),
$$

where $f_{D}=f_{D, d x}$, implies that for $f \in \operatorname{Lip}_{\text {loc }}(\Omega)$,

$$
\begin{aligned}
& \left\|f-f_{D, \rho^{a} d x}\right\|_{L_{\rho}^{p}{ }^{a} d x}(D) \\
& \quad \leq \widetilde{C}\left(r(D)^{(a-b) / p}+\operatorname{diam}(\Omega)^{(a-b) / p}\right) r(D)\|\nabla f\|_{L_{\rho^{b} d x}^{p}(D)},
\end{aligned}
$$

where $f_{D, \rho^{a}} d x=\int_{D} f \rho^{a} d x / \int_{D} \rho^{a} d x$ and $\widetilde{C}$ depends on $C, a, b$ but is independent of $D, f$. To show this, first note that, for such $D$, since $\rho \sim \rho\left(x_{D}\right)$ on $D$, the simple Poincaré estimate immediately gives

$$
\left\|f-f_{D}\right\|_{L_{\rho^{a} d x}^{p}(D)} \leq \widetilde{C} \rho\left(x_{D}\right)^{(a-b) / p} r(D)\|\nabla f\|_{L_{\rho^{b} d x}^{p}(D)}, \quad f \in \operatorname{Lip}_{\mathrm{loc}}(\Omega),
$$

and then a similar estimate with $f_{D}$ replaced by $f_{D, \rho^{a}} d x$ follows by standard arguments. Clearly (2-13) will now follow if we show that

$$
\rho\left(x_{D}\right)^{(a-b) / p} \leq r(D)^{(a-b) / p}+\operatorname{diam}(\Omega)^{(a-b) / p} \text { for such } D .
$$

However, this is clear since $r(D) \leq \rho\left(x_{D}\right) \leq \operatorname{diam}(\Omega)$ for $D$ as above, and (2-13) is proved.

We can now apply the weighted density result of [Hajłasz 1993; Hajłasz and Koskela 1998] to conclude that (2-13) holds for all $f \in L_{\rho^{a} d x}^{1}(\Omega) \cap E_{\rho^{b} d x}^{p}(\Omega)$ and all balls $D$ with $x_{D} \in K$ and $r(D)<\frac{1}{2} \delta_{\epsilon}^{\prime}$.

Recall that $(a-b) / p+1>0$. Thus there exists $r_{\epsilon}$ with $0<r_{\epsilon}<\frac{1}{2} \delta_{\epsilon}^{\prime}$ and

$$
\widetilde{C}\left(r_{\epsilon}^{(a-b) / p}+\operatorname{diam}(\Omega)^{(a-b) / p}\right) r_{\epsilon}<\epsilon .
$$

Let $\Sigma$ and $\Sigma_{0}$ be as in the proof of Theorem 2.1, and let $\left\{E_{l}\right\}_{l}=\left\{F_{l}\right\}_{l}$ be the triples of balls in a maximal collection of pairwise disjoint balls centered in $K$ with radius $\frac{1}{3} r_{\epsilon}$. Then (2-13) and the choice of $r_{\epsilon}$ give the desired version of (1-4), namely

$$
\left\|f-f_{D, \rho^{a} d x}\right\|_{L_{\rho}^{p} a_{d x}(D)} \leq \epsilon\|\nabla f\|_{L_{\rho^{b} d x}^{p}(D)}
$$

for $D=E_{l}$ and $f \in L_{\rho^{a} d x}^{1}(\Omega) \cap E_{\rho^{b} d x}^{p}(\Omega)$. Next, use the last two parts of Remark 2.5 to choose $N \in(q, \infty)$ such that either (2-9) or (2-11) holds with $q$ 
replaced by $N$. Every $f \in L_{\rho^{a} d x}^{1}(\Omega) \cap E_{\rho^{b} d x}^{p}(\Omega)$ then satisfies the global Poincaré estimate

$$
\begin{aligned}
& \left\|f-f_{\Omega, \rho^{a} d x}\right\|_{L_{\rho^{a} d x}^{N}(\Omega)} \leq C\|\nabla f\|_{L_{\rho^{b} d x}^{p}(\Omega)}, \\
& \qquad f \in L_{\rho^{a} d x}^{1}(\Omega) \cap E_{\rho^{b} d x}^{p}(\Omega),
\end{aligned}
$$

where

$$
f_{\Omega, \rho^{a}} d x=\int_{\Omega} f \rho^{a} d x / \int_{\Omega} \rho^{a} d x .
$$

In fact, under the hypothesis of Theorem 2.4, this is proved for example in [Chua and Wheeden 2011] for $f \in \operatorname{Lip}_{\text {loc }}(\Omega) \cap L_{\rho^{a} d x}^{1}(\Omega) \cap E_{\rho^{b} d x}^{p}(\Omega)$, and then follows for all $f \in L_{\rho^{a} d x}^{1}(\Omega) \cap E_{\rho^{b} d x}^{p}(\Omega)$ by the density result of [Hajłasz 1993; Hajłasz and Koskela 1998] and Fatou's lemma. By (2-14),

$$
\|f\|_{L_{\rho^{a} d x}^{N}(\Omega)} \leq C\|f\|_{L_{\rho^{a} a}^{1} d x}(\Omega)+C\|\nabla f\|_{L_{\rho^{b} d x}^{p}(\Omega)}
$$

for the same class of $f$. The remaining details of the proof are left to the reader.

In passing, we mention that the role played by the distance function $\rho\left(x, \Omega^{c}\right)$ in Theorem 2.4 can instead be played by

$$
\rho_{0}(x)=\inf \left\{|x-y|: y \in \Omega_{0}\right\}, \quad x \in \Omega,
$$

for certain $\Omega_{0} \subset \Omega^{c}$; see [Chua and Wheeden 2011, Theorem 1.6] for a description of such $\Omega_{0}$ and the required Poincaré estimate, and note that the density result in [Hajłasz and Koskela 1998] holds for positive continuous weights.

\section{Applications in the degenerate case}

In this section, $\Omega$ will denote a fixed open set in $\mathbb{R}^{n}$, possibly unbounded. For $(x, \xi) \in \Omega \times \mathbb{R}^{n}$, we consider a nonnegative quadratic form $\xi^{\prime} Q(x) \xi$ which may degenerate, that is, which may vanish for some $\xi \neq 0$. Such quadratic forms occur naturally in the context of subelliptic equations and give rise to degenerate Sobolev spaces as discussed below. Our goal is to apply Theorem 1.1 to obtain compact embedding of these degenerate spaces into Lebesgue spaces related to the gain in integrability provided by Poincaré-Sobolev inequalities. The framework that we use contains the subelliptic one developed in [Sawyer and Wheeden 2006; 2010], where regularity theory for weak solutions of linear subelliptic equations of second order in divergence form is studied.

3A. Standing assumptions. We now list some notation and assumptions that will be in force everywhere in Section 3, even when not explicitly mentioned. 
Definition 3.1. A function $d$ is called a finite symmetric quasimetric (or simply a quasimetric) on $\Omega$ if $d: \Omega \times \Omega \rightarrow[0, \infty)$ and there is a constant $\kappa \geq 1$ such that, for all $x, y, z \in \Omega$,

$$
\begin{aligned}
& d(x, y)=d(y, x), \\
& d(x, y)=0 \Longleftrightarrow x=y, \\
& d(x, y) \leq \kappa[d(x, z)+d(z, y)] .
\end{aligned}
$$

If $d$ is a quasimetric on $\Omega$, we refer to the pair $(\Omega, d)$ as a quasimetric space. In some applications, $d$ is closely related to $Q(x)$. For example, $d$ is sometimes chosen to be the Carnot-Carathéodory control metric related to $Q$; cf. [Sawyer and Wheeden 2006].

Given $x \in \Omega, r>0$, and a quasimetric $d$, the subset of $\Omega$ defined by

$$
B_{r}(x)=\{y \in \Omega: d(x, y)<r\}
$$

will be called the quasimetric $d$-ball centered at $x$ of radius $r$. Note that every $d$-ball $B=B_{r}(x)$ satisfies $B \subset \Omega$ by definition.

It is sometimes possible, and desirable in case the boundary of $\Omega$ is rough, to be able to work only with $d$-balls that are deep inside $\Omega$ in the sense that their Euclidean closures $\bar{B}$ lie in $\Omega$. See Remark 3.6(ii) for comments about being able to use such balls.

Recall that $D_{s}(x)$ denotes the ordinary Euclidean ball of radius $s$ centered at $x$. We always assume that $d$ is related to the standard Euclidean metric as follows:

$$
\forall x \in \Omega \text { and } r>0, \exists s=s(x, r)>0 \text { such that } D_{s}(x) \subset B_{r}(x) .
$$

Remark 3.2. Condition (3-2) is clearly true if $d$-balls are open, and it is weaker than the well-known condition of C. Fefferman and Phong stating that for each compact $K \subset \Omega$, there are constants $\beta, r_{0}>0$ such that $D_{r^{\beta}}(x) \subset B_{r}(x)$ for all $x \in K$ and $0<r<r_{0}$.

Throughout Section 3, $Q(x)$ denotes a fixed Lebesgue measurable $n \times n$ nonnegative symmetric matrix on $\Omega$ and we assume that every $d$-ball $B$ centered in $\Omega$ is Lebesgue measurable. We deal with three locally finite measures $w, v, \mu$ on the Lebesgue measurable subsets of $\Omega$, each with a particular role. In Section $3 C$, where only global results are developed, we assume $w(\Omega)<\infty$, but this assumption is not required for the local results of Section 3D. The measure $\mu$ is assumed to be absolutely continuous with respect to Lebesgue measure; the comment following (3-4) explains why this assumption is natural. In Section 3, we sometimes assume that $w$ is absolutely continuous with respect to $v$, but we drop this assumption completely in the Appendix. 
We do not require the existence of a doubling measure for the collection of $d$-balls, but we always assume that $(\Omega, d)$ satisfies the weaker local geometric doubling property given in the next definition; see [Hytönen and Martikainen 2012] for a global version.

Definition 3.3. A quasimetric space $(\Omega, d)$ satisfies the local geometric doubling condition if for every compact $K \subset \Omega$, there exists $\delta^{\prime}=\delta^{\prime}(K)>0$ such that, for all $x \in K$ and all $0<r^{\prime}<r<\delta^{\prime}$, the number of disjoint $d$-balls of radius $r^{\prime}$ contained in $B_{r}(x)$ is at most a constant $\mathscr{C}_{r / r^{\prime}}$ depending on $r / r^{\prime}$ but not on $K$.

3B. The degenerate Sobolev spaces $W_{v, \mu}^{1, p}(\Omega, Q)$ and $W_{v, \mu, 0}^{1, p}(\Omega, Q)$. We will define weighted degenerate Sobolev spaces by using an approach like the one in [Sawyer and Wheeden 2010] or [Monticelli et al. 2012] for the unweighted case. We first define an appropriate space of vectors, including vectors which will eventually play the role of gradients, where size is measured relative to the nonnegative quadratic form

$$
Q(x, \xi)=\xi^{\prime} Q(x) \xi, \quad(x, \xi) \in \Omega \times \mathbb{R}^{n} .
$$

For $1 \leq p<\infty$, consider the collection of measurable $\mathbb{R}^{n}$-valued functions $\vec{g}(x)=$ $\left(g_{1}(x), \ldots, g_{n}(x)\right)$ satisfying

$$
\begin{aligned}
\|\vec{g}\|_{\mathscr{L}_{\mu}^{p}(\Omega, Q)} & =\left\{\int_{\Omega} Q(x, \vec{g}(x))^{p / 2} d \mu\right\}^{1 / p} \\
& =\left\{\int_{\Omega}|\sqrt{Q(x)} \vec{g}(x)|^{p} d \mu\right\}^{1 / p} \\
& <\infty .
\end{aligned}
$$

We identify any two functions $\vec{g}, \vec{h}$ in the collection for which $\|\vec{g}-\vec{h}\|_{\mathscr{L}_{\mu}^{p}(\Omega, Q)}=0$. Then (3-3) defines a norm on the resulting space of equivalence classes. The formweighted space $\mathscr{L}_{\mu}^{p}(\Omega, Q)$ is defined to be the collection of these equivalence classes, with norm (3-3). By using methods similar to those in [Sawyer and Wheeden 2010], it follows that $\mathscr{L}_{\mu}^{2}(\Omega, Q)$ is a Hilbert space and $\mathscr{L}_{\mu}^{p}(\Omega, Q)$ is a Banach space for $1 \leq p<\infty$.

Now consider the (possibly infinite) norm on $\operatorname{Lip}_{\text {loc }}(\Omega)$ defined by

$$
\|f\|_{W_{\nu, \mu}^{1, p}(\Omega, Q)}=\|f\|_{L_{\nu}^{p}(\Omega)}+\|\nabla f\|_{\mathscr{L}_{\mu}^{p}(\Omega, Q)} .
$$

We comment here that our standing assumption that $\mu(Z)=0$ when $Z$ has Lebesgue measure 0 assures that $\|\nabla f\|_{\mathscr{L}_{\mu}^{p}(\Omega, Q)}$ is well-defined if $f \in \operatorname{Lip}_{\text {loc }}(\Omega)$; in fact, for such $f$, the Rademacher-Stepanov theorem implies that $\nabla f$ exists a.e. in $\Omega$ with respect to Lebesgue measure. 
Definition 3.4. Let $1 \leq p<\infty$.

(1) The degenerate Sobolev space $W_{\nu, \mu}^{1, p}(\Omega, Q)$ is the completion under the norm (3-4) of the set

$$
\operatorname{Lip}_{Q, p}(\Omega)=\operatorname{Lip}_{Q, p, v, \mu}(\Omega)=\left\{f \in \operatorname{Lip}_{\text {loc }}(\Omega):\|f\|_{W_{\nu, \mu}^{1, p}(\Omega, Q)}<\infty\right\} .
$$

(2) The degenerate Sobolev space $W_{v, \mu, 0}^{1, p}(\Omega, Q)$ is the completion under the norm (3-4) of the set $\operatorname{Lip}_{Q, p, 0}(\Omega)=\operatorname{Lip}_{0}(\Omega) \cap \operatorname{Lip}_{Q, p}(\Omega)$, where $\operatorname{Lip}_{0}(\Omega)$ denotes the collection of Lipschitz functions with compact support in $\Omega$. If $Q \in L_{\text {loc }}^{p / 2}(\Omega), \operatorname{Lip}_{Q, p, 0}(\Omega)=\operatorname{Lip}_{0}(\Omega)$ since $v$ and $\mu$ are locally finite.

We now make some comments about $W_{\nu, \mu}^{1, p}(\Omega, Q)$, most of which have analogues for $W_{\nu, \mu, 0}^{1, p}(\Omega, Q)$. By definition, $W_{\nu, \mu}^{1, p}(\Omega, Q)$ is the Banach space of equivalence classes of Cauchy sequences of $\operatorname{Lip}_{Q, p}(\Omega)$ functions with respect to the norm (3-4). Given a Cauchy sequence $\left\{f_{j}\right\}$ of $\operatorname{Lip}_{Q, p}(\Omega)$ functions, denote its equivalence class by $\left[\left\{f_{j}\right\}\right]$. If $\left\{v_{j}\right\} \in\left[\left\{f_{j}\right\}\right]$, then $\left\{v_{j}\right\}$ is a Cauchy sequence in $L_{v}^{p}(\Omega)$ and $\left\{\nabla v_{j}\right\}$ is a Cauchy sequence in $\mathscr{L}_{\mu}^{p}(\Omega, Q)$. Hence there is a pair $(f, \vec{g}) \in L_{\nu}^{p}(\Omega) \times \mathscr{L}_{\mu}^{p}(\Omega, Q)$ so that

$$
\left\|v_{j}-f\right\|_{L_{\nu}^{p}(\Omega)} \rightarrow 0 \quad \text { and } \quad\left\|\nabla v_{j}-\vec{g}\right\|_{\mathscr{L}_{\mu}^{p}(\Omega, Q)} \rightarrow 0
$$

as $j \rightarrow \infty$. The pair $(f, \vec{g})$ is uniquely determined by the equivalence class $\left[\left\{f_{j}\right\}\right]$, that is, it is independent of a particular $\left\{v_{j}\right\} \in\left[\left\{f_{j}\right\}\right]$. We say that $(f, \vec{g})$ is represented by $\left\{v_{j}\right\}$. We obtain a Banach space isomorphism $\mathscr{g}$ from $W_{\nu, \mu}^{1, p}(\Omega, Q)$ onto a closed subspace $\mathcal{W}_{\nu, \mu}^{1, p}(\Omega, Q)$ of $L_{\nu}^{p}(\Omega) \times \mathscr{L}_{\mu}^{p}(\Omega, Q)$ by setting

$$
\mathscr{g}\left(\left[\left\{f_{j}\right\}\right]\right)=(f, \vec{g}) .
$$

We often do not distinguish between $W_{\nu, \mu}^{1, p}(\Omega, Q)$ and $\mathcal{W}_{\nu, \mu}^{1, p}(\Omega, Q)$. Similarly, $W_{v, \mu, 0}^{1, p}(\Omega, Q)$ denotes the image of $W_{v, \mu, 0}^{1, p}(\Omega, Q)$ under $\mathscr{F}$, but we often consider these spaces to be the same.

It is important to think of a typical element of $W_{v, \mu}^{1, p}(\Omega, Q)$, or $W_{\nu, \mu}^{1, p}(\Omega, Q)$, as a pair $(f, \vec{g})$ as above, and not simply as the first component $f$. In fact, if $(f, \vec{g}) \in \mathcal{W}_{\nu, \mu}^{1, p}(\Omega, Q)$, the vector $\vec{g}$ may not be uniquely determined by $f$; see [Fabes et al. 1982, Section 2.1] for a well-known example.

If $f \in \operatorname{Lip}_{Q, p}(\Omega)$, the pair $(f, \nabla f)$ may be viewed as an element of $W_{\nu, \mu}^{1, p}(\Omega, Q)$ by identifying it with the equivalence class $[\{f\}]$ corresponding to the sequence each of whose entries is $f$. When viewed as a class, $(f, \nabla f)$ generally contains pairs whose first components are not Lipschitz functions; for example, if $f \in \operatorname{Lip}_{Q, p}(\Omega)$ and $F$ is any function with $F=f$ a.e.- $v$, then $(f, \nabla f)=(F, \nabla f)$ in $W_{v, \mu}^{1, p}(\Omega, Q)$. However, in what follows, when we consider a pair $(f, \nabla f)$ with $f \in \operatorname{Lip}_{Q, p}(\Omega)$, we do not adopt this point of view. Instead we identify an $f \in \operatorname{Lip}_{Q, p}(\Omega)$ with the single pair $(f, \nabla f)$ whose first component is $f$ (defined everywhere in $\Omega$ ) 
and whose second component is $\nabla f$, which exists a.e. with respect to Lebesgue measure by the Rademacher-Stepanov theorem. This convention lets us avoid assuming that $w$ is absolutely continuous with respect to $v$, written $w \ll v$, in Poincaré-Sobolev estimates for $\operatorname{Lip}_{Q, p}(\Omega)$ functions. We reserve the notation $\mathcal{H}$ for subsets of $\operatorname{Lip}_{Q, p}(\Omega)$ viewed in this way.

On the other hand, $W$ denotes various subsets of $W_{v, \mu}^{1, p}(\Omega, Q)$ with elements viewed as equivalence classes. When our hypotheses are phrased in terms of such $W$, we assume that $w \ll v$ in order to avoid technical difficulties associated with sets of measure 0; see the comment after (3-18). In the Appendix, we drop the assumption $w \ll v$ altogether.

We abuse the notation (3-4) by writing

$$
\|(f, \nabla f)\|_{W_{\nu, \mu}^{1, p}(\Omega, Q)}=\|f\|_{L_{v}^{p}(\Omega)}+\|\nabla f\|_{\mathscr{L}_{\mu}^{p}(\Omega, Q)}, \quad f \in \operatorname{Lip}_{Q, p}(\Omega),
$$

and we extend this to generic $(f, \vec{g}) \in W_{\nu, \mu}^{1, p}(\Omega, Q)$ by writing

$$
\|(f, \vec{g})\|_{W_{\nu, \mu}^{1, p}(E, Q)}=\|f\|_{L_{\nu}^{p}(E)}+\|\vec{g}\|_{\mathscr{L}_{\mu}^{p}(E, Q)} \quad \text { for any measurable } E \subset \Omega \text {. }
$$

3C. Global compactness results for degenerate spaces. In this section, we state and prove compactness results which apply to the entire set $\Omega$. Results which are more local are given in Section 3D.

In order to apply Theorem 1.1 in this setting, we use the following version of Poincaré's inequality for $d$-balls.

Definition 3.5. Let $1 \leq p<\infty$, let $\operatorname{Lip}_{Q, p}(\Omega)$ be as in Definition 3.4, and let $\mathscr{H} \subset \operatorname{Lip}_{Q, p}(\Omega)$. We say that the Poincaré property of order $p$ holds for $\mathscr{H}$ if there is a constant $c_{0} \geq 1$ such that for every $\epsilon>0$ and every compact set $K \subset \Omega$, there exists $\delta=\delta(\epsilon, K)>0$ such that, for all $f \in \mathscr{H}$ and every $d$-ball $B_{r}(y)$ with $y \in K$ and $0<r<\delta$,

$$
\left(\int_{B_{r}(y)}\left|f-f_{B_{r}(y), w}\right|^{p} d w\right)^{1 / p} \leq \epsilon\|(f, \nabla f)\|_{W_{v, \mu}^{1, p}\left(B_{c_{0} r}(y), Q\right)} .
$$

Remark 3.6. (i) Inequality (3-8) is not of standard Poincaré form. A more typical form is

$$
\begin{aligned}
& \left(\frac{1}{w\left(B_{r}(y)\right)} \int_{B_{r}(y)}\left|f-f_{B_{r}(y), w}\right|^{p} d w\right)^{1 / p} \\
& \leq C r\left(\frac{1}{\mu\left(B_{c_{0} r}(y)\right)} \int_{B_{c_{0} r}(y)}|\sqrt{Q} \nabla f|^{p} d \mu\right)^{1 / p}
\end{aligned}
$$

for some $c_{0} \geq 1$. In [Sawyer and Wheeden 2006; 2010; Rodney 2007; 2012], the unweighted version of (3-9) with $p=2$ is used. Let $\rho(x, \partial \Omega)$ and $\rho(E, \partial \Omega)$ be as in (2-2). In [Sawyer and Wheeden 2010], the unweighted form of (3-9) with $p=2$ 
is assumed for all $f \in \operatorname{Lip}_{Q, 2}(\Omega)$ and all $B_{r}(y)$ with $y \in \Omega$ and $0<r<\delta_{0} \rho(y, \partial \Omega)$ for some $\delta_{0} \in(0,1)$ independent of $y, r$. If $K$ is a compact set in $\Omega$, this version would then hold for all $B_{r}(y)$ with $y \in K$ and $0<r<\delta_{0} \rho(K, \partial \Omega)$. For general $p$, w, and $\mu$, if for every compact $K \subset \Omega$, (3-9) is valid for all $B_{r}(y)$ with $y \in K$ and $0<r<\delta_{0} \rho(K, \partial \Omega)$, then (3-8) follows easily, provided

$$
\lim _{r \rightarrow 0}\left\{\sup _{y \in K} r^{p} \frac{w\left(B_{r}(y)\right)}{\mu\left(B_{c_{0}}(y)\right)}\right\}=0
$$

for every compact $K \subset \Omega$. Note that (3-10) automatically holds if $w=\mu$.

If both (3-9) and (3-10) hold, then (3-8) is true for any choice of $v$. In this situation, one can pick $v=w$ in order to avoid technicalities encountered below when $w$ is not absolutely continuous with respect to $v$.

(ii) Especially when $\partial \Omega$ is rough, it is simplest to deal only with $d$-balls $B$ which stay away from $\partial \Omega$, that is, which satisfy

$$
\bar{B} \subset \Omega \text {. }
$$

We can always assume this for the balls in (3-8) if the converse of (3-2) is also true, namely, if

$$
\forall x \in \Omega \text { and } r>0, \exists s=s(r, x)>0 \text { such that } B_{S}(x) \subset D_{r}(x) .
$$

To see why, let us first show that given a compact set $K$ and an open set $G$ with $K \subset G \subset \Omega$, there exists $t>0$ so that $\overline{B_{t}(y)} \subset G$ for all $y \in K$. Indeed, for such $K$ and $G$, let $t^{\prime}=\frac{1}{2} \rho\left(K, G^{c}\right)$. By (3-12), for each $x \in K$, there exists $r(x)>0$ such that $B_{r(x)}(x) \subset D_{t^{\prime}}(x)$. Further, by (3-2), there exists $s(x)>0$ such that $D_{s(x)}(x) \subset B_{r(x) /(2 \kappa)}(x)$, where $\kappa$ is as in (3-1). Since $K$ is compact, we may choose finite collections $\left\{B_{r_{i} /(2 \kappa)}\left(x_{i}\right)\right\}$ and $\left\{D_{s_{i}}\left(x_{i}\right)\right\}$ with $x_{i} \in K, r_{i}=r\left(x_{i}\right)$, $s_{i}=s\left(x_{i}\right)$, and $K \subset \bigcup D_{s_{i}}\left(x_{i}\right) \subset \bigcup B_{r_{i} /(2 \kappa)}\left(x_{i}\right)$. Now set $t=\min \left\{r_{i} /(2 \kappa)\right\}$. Let $y \in K$ and choose $i$ such that $y \in B_{r_{i} /(2 \kappa)}\left(x_{i}\right)$. By (3-1), $B_{t}(y) \subset B_{r_{i}}\left(x_{i}\right)$ and, consequently, $B_{t}(y) \subset D_{t^{\prime}}\left(x_{i}\right)$. Since $\overline{D_{t^{\prime}}\left(x_{i}\right)} \subset G$, we obtain $\overline{B_{t}(y)} \subset G$ for every $y \in K$, as desired. In particular, $\overline{B_{t}(y)} \subset \Omega$ for all $y \in K$. Since the validity of (3-8) for some $\delta=\delta(\epsilon, K)$ implies its validity for $\min \{\delta, t\}$, it follows that we may assume (3-11) for every $B_{r}(y)$ in (3-8) when (3-12) holds. Similarly, since the constant $c_{0}$ in (3-8) is independent of $K$, we may also assume that every $B_{c_{0} r}(y)$ in (3-8) has closure in $\Omega$.

(iii) We can often slightly weaken the assumption in Definition 3.5 that $K$ is an arbitrary compact set in $\Omega$. For example, in our results where $w(\Omega)<\infty$, it is generally enough to assume that for each $\epsilon>0$, there is a particular compact $K$ with $w(\Omega \backslash K)<\epsilon$ such that (3-8) holds. However, in Section 3D, where we do not assume $w(\Omega)<\infty$, it is convenient to keep the hypothesis that $K$ is arbitrary. 
Given a set $\mathscr{H} \subset \operatorname{Lip}_{Q, p}(\Omega)$, define

$$
\widehat{\mathscr{H}}=\left\{f: \text { there exists }\left\{f^{j}\right\} \subset \mathscr{H} \text { with } f^{j} \rightarrow f \text { a.e.- } w\right\} .
$$

It will be useful later to note that if $\mathscr{H}$ is bounded in $L_{w}^{N}(\Omega)$ for some $N$, then $\widehat{\mathscr{H}}$ is also bounded in $L_{w}^{N}(\Omega)$ by Fatou's lemma; in particular, every $f \in \widehat{\mathscr{H}}$ then belongs to $L_{w}^{N}(\Omega)$. See (3-15) for a relationship between $\widehat{\mathscr{H}}$ and the closure of $\mathscr{H}$ in $W_{v, \mu}^{1, p}(\Omega, Q)$ in case $w \ll v$.

We now state our simplest global result. Its proof is given after Corollary 3.11.

Theorem 3.7. Let the assumptions of Section $3 A$ hold, $w(\Omega)<\infty, 1 \leq p<\infty$, $1<N \leq \infty$, and $\mathscr{H} \subset \operatorname{Lip}_{Q, p}(\Omega)$. Suppose that the Poincaré property of order $p$ in Definition 3.5 holds for $\mathscr{H}$ and that

$$
\sup _{f \in \mathscr{H}}\left\{\|f\|_{L_{w}^{N}(\Omega)}+\|f\|_{L_{\nu}^{p}(\Omega)}+\|\nabla f\|_{\mathscr{L}_{\mu}^{p}(\Omega, Q)}\right\}<\infty .
$$

Then any sequence $\left\{f_{k}\right\} \subset \widehat{\mathscr{H}}$ has a subsequence that converges in $L_{w}^{q}(\Omega)$ norm for every $1 \leq q<N$ to a function belonging to $L_{w}^{N}(\Omega)$.

Let $\mathscr{H} \subset \operatorname{Lip}_{Q, p}(\Omega)$ and $\widehat{\mathscr{H}}$ be as in (3-13). We reserve the notation $\overline{\mathscr{H}}$ for the closure of $\mathscr{H}$ in $W_{\nu, \mu}^{1, p}(\Omega, Q)$, that is, for the closure of the collection

$$
\{(f, \nabla f): f \in \mathscr{H}\}
$$

with respect to the norm (3-6). Elements of $\overline{\mathscr{H}}$ are viewed as equivalence classes. If $w \ll v$,

$$
\{f: \text { there exists } \vec{g} \text { such that }(f, \vec{g}) \in \overline{\mathscr{H}}\} \subset \widehat{\mathscr{H}} \text {. }
$$

Indeed, if $(f, \vec{g}) \in \overline{\mathscr{H}}$, there is a sequence $\left\{f^{j}\right\} \subset \mathscr{H}$ such that $\left(f^{j}, \nabla f^{j}\right) \rightarrow(f, \vec{g})$ in $W_{\nu, \mu}^{1, p}(\Omega, Q)$ norm, and consequently $f^{j} \rightarrow f$ in $L_{v}^{p}(\Omega)$. By using a subsequence, we may assume that $f^{j} \rightarrow f$ pointwise a.e.- $\nu$, and hence, by absolute continuity, that $f^{j} \rightarrow f$ pointwise a.e.- $w$. This proves (3-15). In fact, it can be verified by using Egorov's theorem that

$$
\left\{f: \text { there exists }\left\{\left(f^{j}, \vec{g}^{j}\right)\right\} \subset \overline{\mathscr{H}} \text { with } f^{j} \rightarrow f \text { a.e.- } w\right\} \subset \widehat{\mathscr{H}} .
$$

Theorem 3.7 and (3-15) immediately imply the following corollary.

Corollary 3.8. Let the assumptions of Section $3 A$ hold, $w(\Omega)<\infty$, and $w \ll v$. Let $1 \leq p<\infty, 1<N \leq \infty, \mathscr{H} \subset \operatorname{Lip}_{Q, p}(\Omega)$, and $\overline{\mathscr{H}}$ be the closure of $\mathscr{H}$ in $W_{v, \mu}^{1, p}(\Omega, Q)$. Suppose that the Poincaré property of order $p$ in Definition 3.5 holds for $\mathscr{H}$ and that

$$
\sup _{f \in \mathscr{H}}\left\{\|f\|_{L_{w}^{N}(\Omega)}+\|(f, \nabla f)\|_{W_{v, \mu}^{1, p}(\Omega, Q)}\right\}<\infty .
$$


Then any sequence $\left\{f_{k}\right\}$ in

$$
\{f \text { : there exists } \vec{g} \text { such that }(f, \vec{g}) \in \overline{\mathscr{H}}\}
$$

has a subsequence that converges in $L_{w}^{q}(\Omega)$ norm for $1 \leq q<N$ to a function that belongs to $L_{w}^{N}(\Omega)$.

Remark 3.9. Corollary 3.8 may be thought of as an analogue in the degenerate setting of the Rellich-Kondrachov theorem since it contains this classical result as a special case. To see why, set $Q(x)=\operatorname{Id}$ and $w=v=\mu$ to be Lebesgue measure. Then, given a bounded sequence $\left\{\left(f_{k}, \vec{g}_{k}\right)\right\} \subset W_{0}^{1, p}(\Omega)=W_{d x, d x, 0}^{1, p}(\Omega, Q)$, we may choose $\left\{f_{k}^{j}\right\} \subset \operatorname{Lip}_{0}(\Omega)$ with $\left(f_{k}^{j}, \nabla f_{k}^{j}\right) \rightarrow\left(f_{k}, \vec{g}_{k}\right)$ in $W^{1, p}(\Omega)$ norm. Thus, setting $\mathscr{H}=\left\{f_{k}^{j}\right\}_{k \in \mathbb{N}, j>J_{k}}$ where each $J_{k}$ is chosen sufficiently large to preserve boundedness, the classical Sobolev inequality gives (3-17) with $N=n p /(n-p)$ for $1 \leq p<n$. The Rellich-Kondrachov theorem now follows from Corollary 3.8.

We next mention analogues of these results when $\mathscr{H}$ is replaced by a set

$$
\mathscr{W} \subset W_{\nu, \mu}^{1, p}(\Omega, Q)
$$

with elements viewed as equivalence classes, assuming that $w \ll v$. We then modify Definition 3.5 by replacing (3-8) with the analogous estimate

$$
\left(\int_{B_{r}(y)}\left|f-f_{B_{r}(y), w}\right|^{p} d w\right)^{1 / p} \leq \epsilon\|(f, \vec{g})\|_{W_{\nu, \mu}^{1, p}\left(B_{c_{0} r}(y), Q\right)} \quad \text { if }(f, \vec{g}) \in \mathcal{W} .
$$

The assumption $w \ll v$ guarantees that the left side of (3-18) does not change when the first component of a pair is arbitrarily altered in a set of $v$-measure zero.

If Poincaré's inequality is known to hold for subsets of Lipschitz functions in the form (3-8), it can often be extended by approximation to the similar form (3-18) for subsets of $W_{\nu, \mu}^{1, p}(\Omega, Q)$. Indeed, let us show without using weak convergence that if $w \ll v$ and the Radon-Nikodym derivative $d w / d v \in L_{v}^{p^{\prime}}(\Omega), 1 / p+1 / p^{\prime}=1$, then (3-18) holds with $\mathcal{W}=W_{\nu, \mu}^{1, p}(\Omega, Q)$ if (3-8) holds with $\mathscr{H}=\operatorname{Lip}_{Q, p}(\Omega)$. This follows easily from Fatou's lemma since if $(f, \vec{g}) \in W_{\nu, \mu}^{1, p}(\Omega, Q)$ and we choose $\left\{f_{j}\right\} \subset \operatorname{Lip}_{Q, p}(\Omega)$ with $\left(f_{j}, \nabla f_{j}\right) \rightarrow(f, \vec{g})$ in $W_{\nu, \mu}^{1, p}(\Omega, Q)$, then, for any ball $B$, since $f_{j} \rightarrow f$ in $L_{v}^{p}(\Omega)$, we have

$$
\left(f_{j}\right)_{B, w}=\frac{1}{w(B)} \int_{B} f_{j} \frac{d w}{d \nu} d \nu \rightarrow \frac{1}{w(B)} \int_{B} f \frac{d w}{d \nu} d v=f_{B, w} .
$$

Of course we may also assume that $f_{j} \rightarrow f$ a.e.- $w$ by selecting a subsequence of $\left\{f_{j}\right\}$ which converges to $f$ a.e.- $v$. The same argument shows that if (3-18) holds for all pairs in any set $\mathscr{W} \subset W_{\nu, \mu}^{1, p}(\Omega, Q)$, then it also holds for pairs in the closure $\bar{W}$ of $\mathcal{W}$ in $W_{\nu, \mu}^{1, p}(\Omega, Q)$. Moreover, if all balls $B$ in question satisfy $\bar{B} \subset \Omega$ (cf. (3-11)), the assumption can clearly be weakened to $d w / d v \in L_{v, \text { loc }}^{p^{\prime}}(\Omega)$. As we observed in 
Remark 3.6(ii), the balls in (3-8) can be assumed to satisfy (3-11) provided (3-12) is true.

Analogues of Theorem 3.7 and Corollary 3.8 for a set $\mathcal{W} \subset W_{\nu, \mu}^{1, p}(\Omega, Q)$ are given in the next result, which also includes the Rellich-Kondrachov theorem as a special case.

Theorem 3.10. Let the assumptions of Section $3 A$ hold, $w(\Omega)<\infty$, and $w \ll v$. Let $1 \leq p<\infty, 1<N \leq \infty$, and $W \subset W_{\nu, \mu}^{1, p}(\Omega, Q)$. Suppose that the Poincaré property in Definition 3.5 holds, but in the modified form given in (3-18), and that

$$
\sup _{(f, \vec{g}) \in \mathcal{W}}\left\{\|f\|_{L_{w}^{N}(\Omega)}+\|(f, \vec{g})\|_{W_{\nu, \mu}^{1, p}(\Omega, Q)}\right\}<\infty .
$$

Let

$$
\widehat{W}=\left\{f \text { : there exists }\left\{\left(f^{j}, \vec{g}^{j}\right)\right\} \subset \mathcal{W} \text { with } f^{j} \rightarrow f \text { a.e. }-w\right\} \text {. }
$$

Then any sequence in $\widehat{W}$ has a subsequence that converges in $L_{w}^{q}(\Omega)$ norm for every $1 \leq q<N$ to a function belonging to $L_{w}^{N}(\Omega)$. In particular, if $\bar{W}$ denotes the closure of $\mathcal{W}$ in $W_{\nu, \mu}^{1, p}(\Omega, Q)$, the same is true for any sequence in

$$
\{f \text { : there exists } \vec{g} \text { such that }(f, \vec{g}) \in \bar{W}\} \text {. }
$$

As a corollary, we obtain a result for arbitrary sequences $\left\{\left(f_{k}, \overrightarrow{g_{k}}\right)\right\}$ which are bounded in $W_{\nu, \mu}^{1, p}(\Omega, Q)$ and whose first components $\left\{f_{k}\right\}$ are bounded in $L_{w}^{N}(\Omega)$.

Corollary 3.11. Let the assumptions of Section $3 A$ hold, $w(\Omega)<\infty, w \ll v$, $1 \leq p<\infty$, and $1<N \leq \infty$. Suppose that the Poincaré property in Definition 3.5 holds for all of $W_{\nu, \mu}^{1, p}(\Omega, Q)$, that is, Definition 3.5 holds with (3-8) replaced by (3-18) for $\mathcal{W}=W_{\nu, \mu}^{1, p}(\Omega, Q)$. Then if $\left\{\left(f_{k}, \vec{g}_{k}\right)\right\}$ is any sequence in $W_{\nu, \mu}^{1, p}(\Omega, Q)$ such that

$$
\sup _{k}\left[\left\|f_{k}\right\|_{L_{w}^{N}(\Omega)}+\left\|\left(f_{k}, \overrightarrow{g_{k}}\right)\right\|_{W_{\nu, \mu}^{1, p}(\Omega, Q)}\right]<\infty,
$$

there is a subsequence of $\left\{f_{k}\right\}$ that converges in $L_{w}^{q}(\Omega)$ norm for $1 \leq q<N$ to a function belonging to $L_{w}^{N}(\Omega)$. If in addition $d w / d v \in L_{v}^{p^{\prime}}(\Omega), 1 / p+1 / p^{\prime}=1$, the conclusion remains valid if the Poincaré property holds just for $\operatorname{Lip}_{Q, p}(\Omega)$.

In fact, the first conclusion in Corollary 3.11 follows by applying Theorem 3.10 with $W$ chosen to be the specific sequence $\left\{\left(f_{k}, \overrightarrow{g_{k}}\right)\right\}_{k}$ in question, and the second statement follows from the first one and our observation above that (3-18) holds with $\mathcal{W}=W_{v, \mu}^{1, p}(\Omega, Q)$ if $d w / d v \in L_{v}^{p^{\prime}}(\Omega), 1 / p+1 / p^{\prime}=1$, and if (3-8) holds with $\mathscr{H}=\operatorname{Lip}_{Q, p}(\Omega)$.

Proofs of Theorems 3.7 and 3.10. We will concentrate on the proof of Theorem 3.7. The proof of Theorem 3.10 is similar and omitted. We begin with a useful covering lemma. 
Lemma 3.12. Let the assumptions of Section $3 A$ hold and let $w(\Omega)<\infty$. Fix $p \in[1, \infty)$ and a set $\mathscr{H} \subset \operatorname{Lip}_{Q, p}(\Omega)$. Suppose the Poincaré property of order $p$ in Definition 3.5 holds for $\mathscr{H}$, and let $\kappa$ be as in (3-1) and $c_{0}$ be as in (3-8). Then, for every $\epsilon>0$, there are positive constants $r=r\left(\epsilon, \kappa, c_{0}\right), M=M\left(\kappa, c_{0}\right)$, and $a$ finite collection $\left\{B_{r}\left(y_{k}\right)\right\}_{k}$ of $d$-balls, such that

$$
\begin{aligned}
& w\left(\Omega \backslash \bigcup_{k} B_{r}\left(y_{k}\right)\right)<\epsilon, \\
& \sum_{k} \chi_{B_{c_{0} r}\left(y_{k}\right)}(x) \leq M \quad \text { for all } x \in \Omega, \\
& \left\|f-f_{B_{r}\left(y_{k}\right), w}\right\|_{L_{w}^{p}\left(B_{r}\left(y_{k}\right)\right)} \leq \epsilon\|(f, \nabla f)\|_{W_{\nu, \mu}^{1, p}\left(B_{c_{0} r}\left(y_{k}\right), Q\right)}
\end{aligned}
$$

for all $f \in \mathscr{H}$ and all $k$. Note that $M$ is independent of $\epsilon$.

Proof. We first recall the "swallowing" property of $d$-balls: there is a constant $\gamma \geq 1$ depending only on $\kappa$ such that if $x, y \in \Omega, 0<r_{1} \leq r_{2}<\infty$ and $B_{r_{1}}(x) \cap B_{r_{2}}(y) \neq \varnothing$, then

$$
B_{r_{1}}(x) \subset B_{\gamma r_{2}}(y) .
$$

Indeed, by [Chua and Wheeden 2008, Observation 2.1], $\gamma$ can be chosen to be $\kappa+2 \kappa^{2}$.

Fix $\epsilon>0$. Since $w(\Omega)<\infty$, there is a compact set $K \subset \Omega$ with $w(\Omega \backslash K)<\epsilon$. Let $\delta^{\prime}=\delta^{\prime}(\epsilon)$ be as in Definition 3.3 for $K$, and let $\delta=\delta(\epsilon)$ be as in (3-8). Fix $r$ with $0<r<\min \left\{\delta, \delta^{\prime} /\left(c_{0} \gamma\right)\right\}$ where $c_{0}$ is as in (3-8). For each $x \in K$, use (3-2) to pick $s(x, r)>0$ so that $D_{s(x, r)}(x) \subset B_{r / \gamma}(x)$. Since $K$ is compact, there are finitely many points $\left\{x_{j}\right\}$ in $K$ such that $K \subset \bigcup_{j} B_{r / \gamma}\left(x_{j}\right)$. Choose a maximal pairwise disjoint subcollection $\left\{B_{r / \gamma}\left(y_{k}\right)\right\}$ of $\left\{B_{r / \gamma}\left(x_{j}\right)\right\}$. We show that the collection $\left\{B_{r}\left(y_{k}\right)\right\}$ satisfies (3-20)-(3-22).

To verify (3-20), it is enough to show that $K \subset \bigcup_{k} B_{r}\left(y_{k}\right)$. Let $y \in K$. Then $y \in B_{r / \gamma}\left(x_{j}\right)$ for some $x_{j}$. If $x_{j}=y_{k}$ for some $y_{k}$ then $y \in B_{r}\left(y_{k}\right)$. If $x_{j} \neq y_{k}$ for all $y_{k}$, there exists $y_{l}$ such that $B_{r / \gamma}\left(y_{l}\right) \cap B_{r / \gamma}\left(x_{j}\right) \neq \varnothing$. Then $B_{r / \gamma}\left(x_{j}\right) \subset B_{r}\left(y_{l}\right)$ by (3-23), and so $y \in B_{r}\left(y_{l}\right)$. In either case, we obtain $y \in \bigcup_{k} B_{r}\left(y_{k}\right)$ as desired.

To verify (3-21), suppose that $\left\{k_{i}\right\}_{i=1}^{L}$ satisfies $\cap_{i=1}^{L} B_{c_{0} r}\left(y_{k_{i}}\right) \neq \varnothing$. Then, by (3-23), $B_{c_{0} r}\left(y_{k_{i}}\right) \subset B_{c_{0} \gamma r}\left(y_{k_{1}}\right)$ for $1 \leq i \leq L$. Since $\gamma, c_{0} \geq 1$, we have

$$
B_{r / \gamma}\left(y_{k}\right) \subset B_{c_{0} r}\left(y_{k}\right)
$$

for all $k$, and consequently

$$
\bigcup B_{r / \gamma}\left(y_{k_{i}}\right) \subset \bigcup B_{c_{0} r}\left(y_{k_{i}}\right) \subset B_{c_{0} \gamma r}\left(y_{k_{1}}\right) .
$$

By construction, $\left\{B_{r / \gamma}\left(y_{k}\right)\right\}$ is pairwise disjoint in $k$. Since $0<r / \gamma<c_{0} \gamma r<\delta^{\prime}$, the corresponding constant $\mathscr{C}$ in the definition of geometric doubling depends only on $\left(c_{0} \gamma r\right) /(r / \gamma)=c_{0} \gamma^{2}$, that is, $\mathscr{C}$ depends only on $\kappa$ and $c_{0}$. Choosing $M$ to be 
this constant, we obtain that $L \leq M$ as desired. The same argument shows that the collection $\left\{B_{c_{0} r}\left(y_{k}\right)\right\}$ has the stronger bounded intercept property with the same bound $M$, that is, any ball in the collection intersects at most $M-1$ others.

Finally, let us verify (3-22). Recall that $0<r<\delta$ by construction. Hence (3-8) implies that for each $k$ and all $f \in \mathscr{H}$,

$$
\left\|f-f_{B_{r}\left(y_{k}\right), w}\right\|_{L_{w}^{p}\left(B_{r}\left(y_{k}\right)\right)} \leq \epsilon\|(f, \nabla f)\|_{W_{\nu, \mu}^{1, p}\left(B_{c_{0} r}\left(y_{k}\right), Q\right)} \cdot
$$

We deduce the proof of Theorem 3.7 from Theorem 1.1 by choosing $\mathscr{X}(\Omega)=$ $L_{\nu}^{p}(\Omega) \times \mathscr{L}_{\mu}^{p}(\Omega, Q)$ and considering the product space

$$
\mathscr{P}_{N, \mathscr{L}(\Omega)}=L_{w}^{N}(\Omega) \times\left(L_{\nu}^{p}(\Omega) \times \mathscr{L}_{\mu}^{p}(\Omega, Q)\right) .
$$

We always choose $\Sigma$ to be the Lebesgue measurable subsets of $\Omega$ and

$$
\Sigma_{0}=\left\{B_{r}(x): r>0, x \in \Omega\right\} .
$$

Note that $\mathscr{L}(\Omega)$ and $\mathscr{B}_{N, \mathscr{L}(\Omega)}$ are normed linear spaces (even Banach spaces), and the norm in $\mathscr{B}_{N, \mathscr{L}(\Omega)}$ is

$$
\|(h,(f, \vec{g}))\|_{\mathscr{B}_{N, \mathscr{Q}(\Omega)}}=\|h\|_{L_{w}^{N}(\Omega)}+\|f\|_{L_{\nu}^{p}(\Omega)}+\|\vec{g}\|_{\mathscr{L}_{\mu}^{p}(\Omega, Q)} .
$$

The roles of $\boldsymbol{g}$ and $(f, \boldsymbol{g})$ in Section 1 are now played by $(f, \vec{g})$ and $(h,(f, \vec{g}))$ respectively.

Let us verify properties (A) and $\left(\mathrm{B}_{p}\right)$ in Section 1 with $\mathscr{L}(\Omega)$ and $\Sigma_{0}$ chosen as above. To verify (A), fix $B \in \Sigma_{0}$ and $(f, \vec{g}) \in \mathscr{X}(\Omega)$. Clearly $f \chi_{B} \in L_{v}^{p}(\Omega)$ since $f \in L_{v}^{p}(\Omega)$. Also,

$$
\begin{aligned}
\int_{\Omega}\left(\left(\vec{g} \chi_{B}\right)^{\prime} Q\left(\vec{g} \chi_{B}\right)\right)^{p / 2} d \mu & =\int_{B}\left(\vec{g}^{\prime} Q(x) \vec{g}\right)^{p / 2} d \mu \\
& \leq \int_{\Omega}\left(\vec{g}^{\prime} Q(x) \vec{g}\right)^{p / 2} d \mu<\infty .
\end{aligned}
$$

Thus $(f, \vec{g}) \chi_{B} \in \mathscr{L}(\Omega)$ and property (A) is proved.

To verify $\left(\mathrm{B}_{p}\right)$, let $\left\{B_{l}\right\}$ be a finite collection of $d$-balls satisfying $\sum_{l} \chi_{B_{l}}(x) \leq C_{1}$ for all $x \in \Omega$. Then if $(f, \vec{g}) \in \mathscr{X}(\Omega)$,

$$
\begin{aligned}
\sum_{l}\left\|(f, \vec{g}) \chi_{B_{l}}\right\|_{\mathscr{L}(\Omega)}^{p} & =\sum_{l}\left(\left\|f \chi_{B_{l}}\right\|_{L_{\nu}^{p}(\Omega)}+\left\|\vec{g} \chi_{B_{l}}\right\|_{\mathscr{L}_{\mu}^{p}(\Omega, Q)}\right)^{p} \\
& \leq 2^{p-1} \sum_{l}\left(\left\|f \chi_{B_{l}}\right\|_{L_{\nu}^{p}(\Omega)}^{p}+\left\|\vec{g} \chi_{B_{l}}\right\|_{\mathscr{L}_{\mu}^{p}(\Omega, Q)}^{p}\right) \\
& =2^{p-1} \int_{\Omega}|f|^{p}\left(\sum_{l} \chi_{B_{l}}\right) d v+\int_{\Omega}\left(\vec{g}^{\prime} Q \vec{g}\right)^{p / 2}\left(\sum_{l} \chi_{B_{l}}\right) d \mu \\
& \leq 2^{p-1} C_{1}\left(\|f\|_{L_{\nu}^{p}(\Omega)}^{p}+\|\vec{g}\|_{\mathscr{L}_{\mu}^{p}(\Omega, Q)}^{p}\right) \leq 2^{p} C_{1}\|(f, \vec{g})\|_{\mathscr{Q}(\Omega)}^{p} .
\end{aligned}
$$


This verifies $\left(\mathrm{B}_{p}\right)$ with $C_{2}$ chosen to be $2^{p} C_{1}$.

The proof of Theorem 3.7 is now very simple. Let $\mathscr{H}$ satisfy its hypotheses and choose $\mathscr{Y}$ in Theorem 1.1 to be the set

$$
\mathscr{S}=\{(f,(f, \nabla f)): f \in \mathscr{H}\} .
$$

Note that $\mathscr{S}$ is a bounded subset of $\mathscr{B}_{N, \mathscr{X}(\Omega)}$ by hypothesis (3-14). Next, in order to choose the pairs $\left\{E_{l}, F_{l}\right\}_{l}$ and verify conditions (i)-(iii) of Theorem 1.1 (see (1-3) and (1-4)), we appeal to Lemma 3.12. Given $\epsilon>0$, let $\left\{E_{l}, F_{l}\right\} l=$ $\left\{B_{r}\left(y_{k}\right), B_{c_{0} r}\left(y_{k}\right)\right\}_{k}$ where $\left\{y_{k}\right\}$ and $r$ are as in Lemma 3.12. Then $E_{l}, F_{l} \in \Sigma_{0}$, and conditions (i)-(iii) of Theorem 1.1 are guaranteed by Lemma 3.12. Finally, by noting that the set $\widehat{\mathscr{H}}$ defined in (3-13) is the same as the set $\hat{\mathscr{S}}$ defined in (1-5), the conclusion of Theorem 3.7 follows from Theorem 1.1.

For special domains $\Omega$ and special choices of $N$, the boundedness assumption (3-14) (or (3-17)) can be weakened to

$$
\sup _{f \in \mathscr{H}}\left\{\|f\|_{L_{\nu}^{p}(\Omega)}+\|\nabla f\|_{\mathscr{L}_{\mu}^{p}(\Omega, Q)}\right\}=\sup _{f \in \mathscr{H}}\|(f, \nabla f)\|_{W_{\nu, \mu}^{1, p}(\Omega, Q)}<\infty .
$$

This is clearly the case for any $\Omega$ and $N$ for which there exists a global SobolevPoincaré estimate that bounds $\|f\|_{L_{w}^{N}(\Omega)}$ by

$$
\|(f, \nabla f)\|_{W_{\nu, \mu}^{1, p}(\Omega, Q)}
$$

for all $f \in \mathscr{H}$. We now formalize this situation assuming that $w \ll v$. In the Appendix, we consider a case when $w \ll v$ fails.

The form of the global Sobolev-Poincaré estimate we will use is given in the next definition. It guarantees that (3-14) and (3-26) are the same when $N=p \sigma$.

Definition 3.13. Let $1 \leq p<\infty$ and $\mathscr{H} \subset \operatorname{Lip}_{Q, p}(\Omega)$. Then the global Sobolev property of order $p$ holds for $\mathscr{H}$ if there are constants $C>0$ and $\sigma>1$ such that

$$
\|f\|_{L_{w}^{p \sigma}(\Omega)} \leq C\|(f, \nabla f)\|_{W_{\nu, \mu}^{1, p}(\Omega, Q)} \text { for all } f \in \mathcal{H} .
$$

If $w \ll v,(3-27)$ extends to $(f, \vec{g}) \in \overline{\mathscr{H}}$. In fact, let $(f, \vec{g}) \in \overline{\mathscr{H}}$ and choose $\left\{f_{j}\right\} \subset \mathscr{H}$ with $\left(f_{j}, \nabla f_{j}\right) \rightarrow(f, \vec{g})$ in $W_{\nu, \mu}^{1, p}(\Omega, Q)$. Then $f_{j} \rightarrow f$ in $L_{\nu}^{p}(\Omega)$ norm, and by choosing a subsequence we may assume that $f_{j} \rightarrow f$ a.e.- $v$. Hence $f_{j} \rightarrow f$ a.e.- $w$ because $w \ll v$. Since each $f_{j}$ satisfies (3-27), it follows that

$$
\|f\|_{L_{w}^{p \sigma}(\Omega)} \leq C\|(f, \vec{g})\|_{W_{\nu, \mu}^{1, p}(\Omega, Q)} \quad \text { if }(f, \vec{g}) \in \overline{\mathscr{H}} .
$$

Under the same assumptions, namely, that Definition 3.13 holds for a set

$$
\mathscr{H} \subset \operatorname{Lip}_{Q, p}(\Omega)
$$


and that $w \ll v$, the same sequence $\left\{f_{j}\right\}$ as above is also bounded in $L_{w}^{p \sigma}(\Omega)$ norm and so satisfies $\left(f_{j}\right)_{E, w} \rightarrow f_{E, w}$ for measurable $E$ by the same weak convergence argument given after the statement of Theorem 1.1. Hence the Poincare estimate in Definition 3.5 also extends to $\overline{\mathscr{H}}$ in the same form as (3-18), with $\mathcal{W}$ replaced by $\overline{\mathscr{H}}$, that is,

$$
\left(\int_{B_{r}(y)}\left|f-f_{B_{r}(y), w}\right|^{p} d w\right)^{1 / p} \leq \epsilon\|(f, \vec{g})\|_{W_{\nu, \mu}^{1, p}\left(B_{c_{0} r}(y), Q\right)} \text { if }(f, \vec{g}) \in \overline{\mathcal{H}} .
$$

Hence we immediately obtain the next result by choosing $\mathscr{W}=\overline{\mathscr{H}}$ and $N=p \sigma$ in Theorem 3.10.

Theorem 3.14. Let the assumptions of Section $3 A$ hold, $w(\Omega)<\infty$, and $w \ll v$. Fix $p \in[1, \infty)$ and a set $\mathscr{H} \subset \operatorname{Lip}_{Q, p}(\Omega)$. Suppose the Poincaré and global Sobolev properties of order $p$ in Definitions 3.5 and 3.13 hold for $\mathscr{H}$, and let $\sigma$ be as in (3-27). If $\left\{\left(f_{k}, \vec{g}_{k}\right)\right\}$ is a sequence in $\overline{\mathcal{H}}$ with

$$
\sup _{k}\left\|\left(f_{k}, \overrightarrow{g_{k}}\right)\right\|_{W_{\nu, \mu}^{1, p}(\Omega, Q)}<\infty,
$$

then $\left\{f_{k}\right\}$ has a subsequence which converges in $L_{w}^{q}(\Omega)$ for $1 \leq q<p \sigma$, and the limit of the subsequence belongs to $L_{w}^{p \sigma}(\Omega)$.

A result for the entire space $W_{\nu, \mu}^{1, p}(\Omega, Q)$ follows by choosing $\mathscr{H}=\operatorname{Lip}_{Q, p}(\Omega)$ in Theorem 3.14 or Corollary 3.8:

Corollary 3.15. Suppose the hypotheses of Theorem 3.14 hold with $\mathscr{H}=\operatorname{Lip}_{Q, p}(\Omega)$. If $\left\{\left(f_{k}, \overrightarrow{g_{k}}\right)\right\} \subset W_{\nu, \mu}^{1, p}(\Omega, Q)$ and (3-30) is true, $\left\{f_{k}\right\}$ has a subsequence which converges in $L_{w}^{q}(\Omega)$ for $1 \leq q<p \sigma$, and the limit of the subsequence belongs to $L_{w}^{p \sigma}(\Omega)$.

See the Appendix for analogues of Theorem 3.14 and Corollary 3.15 without the assumption $w \ll v$.

3D. Local compactness results for degenerate spaces. In this section, for general bounded open sets $\Omega^{\prime}$ with $\bar{\Omega}^{\prime} \subset \Omega$, we study compact embedding of subsets of $W_{\nu, \mu}^{1, p}(\Omega, Q)$ into $L_{w}^{q}\left(\Omega^{\prime}\right)$ without assuming a global Sobolev estimate for $\Omega$ or $\Omega^{\prime}$ and without assuming $w(\Omega)<\infty$. For some applications, see the comment at the end of the section.

The theorems below assume a much weaker condition than the global Sobolev estimate (3-27), namely, the following local estimate.

Definition 3.16. Let $1 \leq p<\infty$. We say that the local Sobolev property of order $p$ holds if, for some fixed constant $\sigma>1$ and every compact set $K \subset \Omega$, there is a constant $r_{1}>0$ such that, for all $d$-balls $B=B_{r}(y)$ with $y \in K$ and $0<r<r_{1}$,

$$
\|f\|_{L_{w}^{p \sigma}(B)} \leq C(B)\|(f, \nabla f)\|_{W_{\nu, \mu}^{1, p}(\Omega, Q)} \text { if } f \in \operatorname{Lip}_{0}(\Omega) \text { with supp } f \subset B,
$$


where $C(B)$ is a positive constant independent of $f$.

Remark 3.17. (i) A more standard assumption than (3-31) is a normalized inequality that includes a factor $r$ in the gradient term on the right side:

$$
\left(\frac{1}{w\left(B_{r}(y)\right)} \int_{B_{r}(y)}|f|^{p \sigma} d w\right)^{1 /(p \sigma)}
$$

$$
\leq C\left(\frac{1}{v\left(B_{r}(y)\right)} \int_{B_{r}(y)}|f|^{p} d \nu\right)^{1 / p}+C r\left(\frac{1}{\mu\left(B_{r}(y)\right)} \int_{B_{r}(y)}|\sqrt{Q} \nabla f|^{p} d \mu\right)^{1 / p},
$$

with $C$ independent of $r, y$; see, for example, [Sawyer and Wheeden 2006; Rodney $2007 ; 2012]$ in the unweighted case with $p=2$. Clearly (3-32) is a stronger requirement than (3-31).

(ii) In the classical $n$-dimensional elliptic case for linear second order equations in divergence form, $Q$ satisfies $c|\xi|^{2} \leq Q(x, \xi) \leq C|\xi|^{2}$ for some fixed constants $c, C>0$ and $d$ is the standard Euclidean metric $d(x, y)=|x-y|$. For $1 \leq p<n$ and $\sigma=n /(n-p),(3-31)$ then holds with $d w=d v=d \mu=d x$ since the corresponding version of (3-32) is true with $|\sqrt{Q} \nabla f|$ replaced by $|\nabla f|$.

We also use a notion of Lipschitz cutoff functions on $d$-balls:

Definition 3.18. For $s \geq 1$, we say that the cutoff property of order $s$ holds for $\mu$ if, for each compact $K \subset \Omega$, there exists $\delta=\delta(K)>0$ such that, for every d-ball $B_{r}(y)$ with $y \in K$ and $0<r<\delta$, there is a function $\phi \in \operatorname{Lip}_{0}(\Omega)$ and a constant $\gamma=\gamma(y, r) \in(0, r)$ satisfying

(i) $0 \leq \phi \leq 1$ in $\Omega$,

(ii) $\operatorname{supp} \phi \subset B_{r}(y)$ and $\phi=1$ in $B_{\gamma}(y)$,

(iii) $\nabla \phi \in \mathscr{L}_{\mu}^{s}(\Omega, Q)$.

Since $\mu$ is always assumed to be locally finite, the strongest form of Definition 3.18, namely, the version with $s=\infty$, automatically holds if $Q$ is locally bounded in $\Omega$ and (3-12) is true; recall that we always assume (3-2). To see why, fix a compact set $K \subset \Omega$ and consider $B_{r}(y)$ with $y \in K$ and $r<1$. Use (3-2) to choose open Euclidean balls $D^{\prime}, D$ with common center $y$ such that $\bar{D}^{\prime} \subset D \subset B_{r}(y)(\subset \Omega$ by definition). Construct a smooth function $\phi$ in $\Omega$ with support in $D$ such that $0 \leq \phi \leq 1$ and $\phi=1$ on $D^{\prime}$. By (3-12), there is $\gamma>0$ such that $B_{\gamma}(y) \subset D^{\prime}$. Then $\phi$ satisfies Definition 3.18(i)-(iii) with $s=\infty$; for (iii), we use the fact that $\nabla \phi$ has compact support in $\Omega$ together with local boundedness of $Q$ and local finiteness of $\mu$.

To compensate for the lack of a global Sobolev estimate, given $\mathscr{H} \subset \operatorname{Lip}_{Q, p}(\Omega)$, we assume in conjunction with the cutoff property of some order $s \geq p \sigma^{\prime}$ that, for every compact set $K \subset \Omega$, there exists $\delta=\delta(K)>0$ such that, for every $d$-ball $B$ 
with center in $K$ and radius less than $\delta$, there is a constant $C_{1}(B)$ such that

$$
\|f\|_{L_{\mu}^{p t^{\prime}(B)}} \leq C_{1}(B)\|(f, \nabla f)\|_{W_{v, \mu}^{1, p}(\Omega, Q)} \quad \text { if } f \in \mathscr{H},
$$

where $t=s / p$ and $1 / t+1 / t^{\prime}=1$. Note that $1 \leq t^{\prime} \leq \sigma$ since $s \geq p \sigma^{\prime}$.

Remark 3.19. Inequality (3-33) is different in nature from (3-31) even if $t^{\prime}=\sigma$ and $w=\mu$ since there is a restriction on supports in (3-31) but not in (3-33). However, (3-33) implies (3-31) when $s=p \sigma^{\prime}, w=\mu$, and $\mathscr{H}$ contains all functions in $\operatorname{Lip}_{0}(\Omega)$ with support in any ball. On the other hand, (3-33) is often automatic if $\mu=v$. For example, as mentioned earlier, if $Q$ is locally bounded and (3-12) is true, the cutoff property holds with $s=\infty$, giving $t=\infty$ and $t^{\prime}=1$. In this case, when $\mu=v$, the left side of (3-33) is clearly smaller than the right side (in fact smaller than $\|f\|_{L_{\nu}^{p}(\Omega)}$ ).

We can now state our main local result.

Theorem 3.20. Let the assumptions of Section $3 A$ and condition (3-12) hold, and let $w \ll v$. Fix $p \in[1, \infty)$ and suppose the Poincaré property of order $p$ in Definition 3.5 holds for a fixed set $\mathcal{H} \subset \operatorname{Lip}_{Q, p}(\Omega)$ and the local Sobolev property of order $p$ in Definition 3.16 holds. Assume the cutoff property of some order $s \geq p \sigma^{\prime}$ is true for $\mu$, with $\sigma$ as in (3-31), and that (3-33) holds for He with $t=s / p$. Then, for every $\left\{\left(f_{k}, \overrightarrow{g_{k}}\right)\right\} \subset \overline{\mathscr{H}}$ that is bounded in $W_{v, \mu}^{1, p}(\Omega, Q)$ norm, there is a subsequence $\left\{f_{k_{i}}\right\}$ of $\left\{f_{k}\right\}$ and an $f \in L_{w, \text { loc }}^{p \sigma}(\Omega)$ such that $f_{k_{i}} \rightarrow f$ pointwise a.e.- $w$ in $\Omega$ and in $L_{w}^{q}\left(\Omega^{\prime}\right)$ norm for all $1 \leq q<p \sigma$ and every bounded open $\Omega^{\prime}$ with $\bar{\Omega}^{\prime} \subset \Omega$.

See the Appendix for a version of Theorem 3.20 without assuming $w \ll v$.

Recall that $\overline{\mathscr{H}}=W_{\nu, \mu}^{1, p}(\Omega, Q)$ if $\mathscr{H}=\operatorname{Lip}_{Q, p}(\Omega)$. In the important case when $Q \in L_{\mathrm{loc}}^{\infty}(\Omega)$, Theorem 3.20 and Remark 3.19 immediately imply the next result.

Corollary 3.21. Let $Q$ be locally bounded in $\Omega$ and suppose that (3-12) holds. Fix $p \in[1, \infty)$, and with $w=v=\mu$, assume the Poincaré property of order $p$ holds for $\operatorname{Lip}_{Q, p}(\Omega)$ and the local Sobolev property of order $p$ holds. Then, for every bounded sequence $\left\{\left(f_{k}, \vec{g}_{k}\right)\right\} \subset W_{w, w}^{1, p}(\Omega, Q)$, there is a subsequence $\left\{f_{k_{i}}\right\}$ of $\left\{f_{k}\right\}$ and a function $f \in L_{w, \mathrm{loc}}^{p \sigma}(\Omega)$ such that $f_{k_{i}} \rightarrow f$ pointwise a.e.- $w$ in $\Omega$ and in $L_{w}^{q}\left(\Omega^{\prime}\right)$ norm, $1 \leq q<p \sigma$, for every bounded open $\Omega^{\prime}$ with $\bar{\Omega}^{\prime} \subset \Omega$.

Proof of Theorem 3.20. We begin by using the cutoff property in Definition 3.18 to construct a partition of unity relative to $d$-balls and compact subsets of $\Omega$.

Lemma 3.22. Fix $\Omega$ and $s \geq 1$, and suppose the cutoff property of order $s$ holds for $\mu$. If $K$ is a compact subset of $\Omega$ and $r>0$, there is a finite collection of $d$-balls $\left\{B_{r}\left(y_{j}\right)\right\}$ with $y_{j} \in K$ together with functions $\left\{\psi_{j}\right\}$ in $\operatorname{Lip}_{0}(\Omega)$ such that $\operatorname{supp} \psi_{j} \subset B_{r}\left(y_{j}\right)$ and 
(a) $K \subset \bigcup_{j} B_{r}\left(y_{j}\right)$,

(b) $0 \leq \psi_{j} \leq 1$ in $\Omega$ for each $j$ and $\sum_{j} \psi_{j}(x)=1$ for all $x \in K$,

(c) $\nabla \psi_{j} \in \mathscr{L}_{\mu}^{s}(\Omega, Q)$ for each $j$.

Proof. The argument is an adaptation of one in [Rudin 1987] for the usual Euclidean case. The authors thank D. D. Monticelli for related discussions. Fix $r>0$ and a compact set $K \subset \Omega$, and set $\beta=\min \{\delta / 2, r\}$ for $\delta=\delta(K)$ as in Definition 3.18. Since $\beta<\delta$, Definition 3.18 implies that, for each $y \in K$, there exist $\gamma(y) \in(0, \beta)$ and $\phi_{y}(x) \in \operatorname{Lip}_{0}(\Omega)$ such that $0 \leq \phi_{y} \leq 1$ in $\Omega$, supp $\left.\phi_{y} \subset B_{\beta}(y)\right), \phi_{y}=1$ in $B_{\gamma(y)}(y)$ and $\nabla \phi_{y} \in \mathscr{L}_{\mu}^{s}(\Omega, Q)$. The collection $\left\{B_{\gamma(y)}(y)\right\}_{y \in K}$ covers $K$, so by (3-2) and the compactness of $K$, there is a finite subcollection $\left\{B_{\gamma}\left(y_{j}\right)\left(y_{j}\right)\right\}_{j=1}^{m}$ whose union covers $K$. Part (a) follows since $\gamma\left(y_{j}\right)<r$. Next let $\phi_{j}(x)=\phi_{y_{j}}(x)$ and define $\left\{\psi_{j}\right\}_{j=1}^{m}$ as follows: set $\psi_{1}=\phi_{1}$ and

$$
\psi_{j}=\left(1-\phi_{1}\right) \cdots\left(1-\phi_{j-1}\right) \phi_{j}
$$

for $j=2, \ldots, m$. Then each $\psi_{j} \in \operatorname{Lip}_{0}(\Omega)$, and supp $\phi_{j} \subset B_{r}\left(y_{j}\right)$ since $\beta<r$. Also, $0 \leq \psi_{j} \leq 1$ in $\Omega$ and

$$
\sum_{j=1}^{m} \psi_{j}(x)=1-\prod_{j=1}^{m}\left(1-\phi_{j}(x)\right), \quad x \in \Omega .
$$

If $x \in K, x \in B_{\gamma\left(y_{j}\right)}\left(y_{j}\right)$ for some $j$. Hence some $\phi_{j}(x)=1$ and consequently $\sum_{j} \psi_{j}(x)=1$. This proves part (b). Lastly, we use Leibniz's product rule to compute $\nabla \psi_{j}$ and then apply Minkowski's inequality $j$ times to obtain part (c) from the fact that $\nabla \phi_{j} \in \mathscr{L}_{\mu}^{s}(\Omega, Q)$.

The next lemma shows how the local Sobolev estimate (3-31) and Lemma 3.22 lead to a local analogue of the global Sobolev estimate (3-27).

Lemma 3.23. Let $\Omega^{\prime}$ be a bounded open set with $\bar{\Omega}^{\prime} \subset \Omega$. Suppose that both Definition 3.16 and the cutoff property for $\mu$ of some order $s \geq p \sigma^{\prime}$ hold, and also that (3-33) holds with $t=s / p$ for a fixed set $\mathscr{H} \subset \operatorname{Lip}_{\text {loc }}(\Omega)$. Then there is a finite constant $C\left(\Omega^{\prime}\right)$ such that

$$
\|f\|_{L_{w}^{p \sigma}\left(\Omega^{\prime}\right)} \leq C\left(\Omega^{\prime}\right)\|(f, \nabla f)\|_{W_{\nu, \mu}^{1, p}(\Omega, Q)} \quad \text { if } f \in \mathscr{H} .
$$

Proof. Let $r_{1}$ be as in Definition 3.16 relative to the compact set $\bar{\Omega}^{\prime} \subset \Omega$, and let $\delta$ be as in (3-33). Use Lemma 3.22 to cover $\bar{\Omega}^{\prime}$ by the union of a finite number of $d$-balls $\left\{B_{j}\right\}$ each of radius smaller than $\min \left\{r_{1}, \delta\right\}$. Associated with this cover is a collection $\left\{\psi_{j}\right\} \subset \operatorname{Lip}_{0}(\Omega)$ with $\operatorname{supp} \psi_{j} \subset B_{j}, \sum_{j} \psi_{j}=1$ in $\Omega^{\prime}$, and

$$
\nabla \psi_{j} \in \mathscr{L}_{\mu}^{s}(\Omega, Q) .
$$


If $f \in \mathscr{H}$, then

$$
\|f\|_{L_{w}^{p \sigma}\left(\Omega^{\prime}\right)}=\left\|f \sum_{j} \psi_{j}\right\|_{L_{w}^{p \sigma}\left(\Omega^{\prime}\right)} \leq \sum_{j}\left\|\psi_{j} f\right\|_{L_{w}^{p \sigma}\left(B_{j}\right)} .
$$

Since $\psi_{j} f \in \operatorname{Lip}_{0}(\Omega)$ and $\operatorname{supp}\left(\psi_{j} f\right) \subset B_{j}$, the estimate (3-31) and the product rule give

$$
\begin{aligned}
& \left\|\psi_{j} f\right\|_{L_{w}^{p \sigma}\left(B_{j}\right)} \\
& \leq C\left(B_{j}\right)\left\|\left(\psi_{j} f, \nabla\left(\psi_{j} f\right)\right)\right\|_{W_{\nu, \mu}^{1, p}\left(B_{j}, Q\right)} \\
& =C\left(B_{j}\right)\left(\left\|\psi_{j} f\right\|_{L_{\nu}^{p}\left(B_{j}\right)}+\left\|\sqrt{Q} \nabla\left(\psi_{j} f\right)\right\|_{L_{\mu}^{p}\left(B_{j}\right)}\right) \\
& \leq C\left(B_{j}\right)\left(\left\|\psi_{j} f\right\|_{L_{\nu}^{p}\left(B_{j}\right)}+\left\|\psi_{j} \sqrt{Q} \nabla f\right\|_{L_{\mu}^{p}\left(B_{j}\right)}+\left\|f \sqrt{Q} \nabla \psi_{j}\right\|_{L_{\mu}^{p}\left(B_{j}\right)}\right) \\
& \leq C\left(B_{j}\right)\left(\|(f, \nabla f)\|_{W_{\nu, \mu}^{1, p}(\Omega, Q)}+\left\|f \sqrt{Q} \nabla \psi_{j}\right\|_{L_{\mu}^{p}\left(B_{j}\right)}\right),
\end{aligned}
$$

where we have used $\left|\psi_{j}\right| \leq 1$. We estimate the second term on the right of (3-36) by using (3-33). Recall that $t=s / p \geq \sigma^{\prime}$ and $1 / t+1 / t^{\prime}=1$. Let

$$
\bar{C}=\max _{j}\left\|\sqrt{Q} \nabla \psi_{j}\right\|_{L_{\mu}^{s}\left(B_{j}\right)}
$$

By Hölder's inequality and (3-33),

$$
\begin{aligned}
\left\|f \sqrt{Q} \nabla \psi_{j}\right\|_{L_{\mu}^{p}\left(B_{j}\right)} & \leq\|f\|_{L_{\mu}^{p t^{\prime}\left(B_{j}\right)}}\left\|\sqrt{Q} \nabla \psi_{j}\right\|_{L_{\mu}^{s}\left(B_{j}\right)} \\
& \leq \bar{C} C_{1}\left(B_{j}\right)\|(f, \nabla f)\|_{W_{\nu, \mu}^{1, p}(\Omega, Q)}
\end{aligned}
$$

Combining this with (3-36) gives

$$
\left\|\psi_{j} f\right\|_{L_{w}^{p \sigma}\left(B_{j}\right)} \leq C\left(B_{j}\right)\left(1+\bar{C} C_{1}\left(B_{j}\right)\right)\|(f, \nabla f)\|_{W_{\nu, \mu}^{1, p}(\Omega, Q)} .
$$

By (3-35), for any $f \in \mathscr{H}$,

$$
\begin{aligned}
\|f\|_{L_{w}^{p \sigma}\left(\Omega^{\prime}\right)} & \leq\|(f, \nabla f)\|_{W_{\nu, \mu}^{1, p}(\Omega, Q)} \sum_{j} C\left(B_{j}\right)\left(1+\bar{C} C_{1}\left(B_{j}\right)\right) \\
& =C\left(\Omega^{\prime}\right)\|(f, \nabla f)\|_{W_{\nu, \mu}^{1, p}(\Omega, Q)} .
\end{aligned}
$$

Theorem 3.20 follows from Lemma 3.23 and Theorem 1.4. We sketch the proof, omitting some familiar details. By choosing a sequence of compact sets increasing to $\Omega$ and using a diagonalization argument, it is enough to prove the conclusion for a fixed open $\Omega^{\prime}$ with compact closure $\bar{\Omega}^{\prime}$ in $\Omega$. Fix such an $\Omega^{\prime}$ and select a bounded open $\Omega^{\prime \prime}$ with $\bar{\Omega}^{\prime} \subset \Omega^{\prime \prime} \subset \bar{\Omega}^{\prime \prime} \subset \Omega$. For $\mathscr{H}$ as in Theorem 3.20, apply Lemma 3.23 to the set $\Omega^{\prime \prime}$ to obtain

$$
\|f\|_{L_{w}^{p \sigma}\left(\Omega^{\prime \prime}\right)} \leq C\left(\Omega^{\prime \prime}\right)\|(f, \nabla f)\|_{W_{\nu, \mu}^{1, p}(\Omega, Q)}, \quad f \in \mathscr{H} .
$$


By assumption, $w \ll v$, so (3-38) extends to $\overline{\mathscr{H}}$ in the form

$$
\|f\|_{L_{w}^{p \sigma}\left(\Omega^{\prime \prime}\right)} \leq C\left(\Omega^{\prime \prime}\right)\|(f, \vec{g})\|_{W_{\nu, \mu}^{1, p}(\Omega, Q)}, \quad(f, \vec{g}) \in \overline{\mathscr{H}} .
$$

Let $\epsilon>0$. By hypothesis, $\mathscr{H}$ satisfies the Poincaré estimate (3-8) for balls $B_{r}(y)$ with $y \in \bar{\Omega}^{\prime}$ and $r<\delta\left(\epsilon, \Omega^{\prime}\right)$. Since the Euclidean distance between $\bar{\Omega}^{\prime}$ and $\partial \Omega^{\prime \prime}$ is positive and we have assumed (3-12), we may also assume by Remark 3.6(ii) that all such balls lie in the larger set $\Omega^{\prime \prime}$. Next we claim that (3-8) extends to $\overline{\mathscr{H}}$, that is,

$$
\text { (3-40) }\left(\int_{B_{r}(y)}\left|f-f_{B_{r}(y), w}\right|^{p} d w\right)^{1 / p} \leq \epsilon\|(f, \vec{g})\|_{W_{\nu, \mu}^{1, p}\left(B_{c_{0} r}(y), Q\right)} \text { if }(f, \vec{g}) \in \overline{\mathscr{H}},
$$

for the same class of balls $B_{r}(y)$. In fact, if $(f, \vec{g}) \in \overline{\mathscr{H}}$ and $\left\{f^{j}\right\} \subset \mathscr{H}$ satisfies $\left(f^{j}, \nabla f^{j}\right) \rightarrow(f, \vec{g})$ in $W_{\nu, \mu}^{1, p}(\Omega, Q)$ norm, then there is a subsequence, still denoted $\left\{f^{j}\right\}$, with $f^{j} \rightarrow f$ a.e.- $v$ in $\Omega$, and so with $f^{j} \rightarrow f$ a.e.- $w$ in $\Omega$ since $w \ll v$. By (3-38), $\left\{f^{j}\right\}$ is bounded in $L_{w}^{p \sigma}\left(\Omega^{\prime \prime}\right)$. Hence, since the balls in (3-40) satisfy $B_{r}(y) \subset \Omega^{\prime \prime}$, we obtain $f_{B_{r}(y), w}^{j} \rightarrow f_{B_{r}(y), w}$ by our usual weak convergence argument, and (3-40) follows by Fatou's lemma from its analogue (3-8) for the $\left(f^{j}, \nabla f^{j}\right)$.

Now let $\left\{\left(f_{k}, \overrightarrow{g_{k}}\right)\right\} \subset \overline{\mathscr{H}}$ be bounded in $W_{\nu, \mu}^{1, p}(\Omega, Q)$ norm and apply Theorem 1.4 with $\mathscr{X}(\Omega)=L_{\nu}^{p}(\Omega) \times \mathscr{L}_{\mu}^{p}(\Omega, Q)$ to the set $\mathscr{S}$ defined by

$$
\mathscr{S}=\left\{\left(f_{k},\left(f_{k}, \overrightarrow{g_{k}}\right)\right)\right\}_{k},
$$

and with $\left\{\left(E_{l}^{\epsilon}, F_{l}^{\epsilon}\right)\right\}_{l}$ chosen to be a finite number of pairs $\left\{\left(B_{r}\left(y_{l}\right), B_{c_{0}}\left(y_{l}\right)\right\}_{l}\right.$ as in (3-40), but now with $r$ fixed depending on $\epsilon$, and with $\Omega^{\prime} \subset \bigcup_{l} B_{r}\left(y_{l}\right)$. Such a finite choice exists by (3-2) and the Heine-Borel theorem since $\bar{\Omega}^{\prime}$ is compact; cf. the proof of Lemma 3.12. Since $\Omega^{\prime}$ is completely covered by $\bigcup_{l} E_{l}^{\epsilon}$, assumption (i) of Theorem 1.4 is fulfilled. Moreover, the collection $\left\{F_{l}^{\epsilon}\right\}$ has bounded overlaps uniformly in $\epsilon$ by the geometric doubling argument used to prove Lemma 3.12.

Finally, (1-15) follows from (3-39) applied to the bounded sequence $\left\{\left(f_{k}, \vec{g}_{k}\right)\right\}$ since $\bigcup_{l, \epsilon} E_{l}^{\epsilon} \subset \Omega^{\prime \prime}$. Thus Theorem 1.4 implies that there is a subsequence $\left\{f_{k_{i}}\right\}$ of $\left\{f_{k}\right\}$ and a function $f \in L_{w}^{p \sigma}\left(\Omega^{\prime}\right)$ such that $f_{k_{i}} \rightarrow f$ a.e.- $w$ in $\Omega^{\prime}$ and in $L_{w}^{q}\left(\Omega^{\prime}\right)$ norm, $1 \leq q<p \sigma$. This completes the proof of Theorem 3.20.

For functions which are compactly supported in a fixed bounded open $\Omega^{\prime}$ with $\bar{\Omega}^{\prime} \subset \Omega$, the proof of Theorem 3.20 can be modified to yield compact embedding into $L_{w}^{q}\left(\Omega^{\prime}\right)$ for the same $\Omega^{\prime}$ without assuming (3-12). Of course we always require (3-2). Given such $\Omega^{\prime}$ and a set $\mathcal{H} \subset \operatorname{Lip}_{Q, p, 0}\left(\Omega^{\prime}\right)$, we may view $\mathcal{H}$ as a subset of $\operatorname{Lip}_{Q, p, 0}(\Omega)$ simply by extending functions in $\mathcal{H}$ to all of $\Omega$ as 0 in $\Omega \backslash \Omega^{\prime}$. In this way, the proof of Theorem 3.20 works without (3-12). For example, choosing $\mathscr{H}=\operatorname{Lip}_{Q, p, 0}\left(\Omega^{\prime}\right)$, we obtain: 
Theorem 3.24. Let the assumptions of Section $3 A$ hold and $w \ll v$. Let $\Omega^{\prime}$ be a bounded open set with $\bar{\Omega}^{\prime} \subset \Omega$. Fix $p \in[1, \infty)$ and suppose the Poincaré property of order $p$ in Definition 3.5 holds for $\operatorname{Lip}_{Q, p, 0}\left(\Omega^{\prime}\right)$, with $\operatorname{Lip}_{Q, p, 0}\left(\Omega^{\prime}\right)$ viewed as a subset of $\operatorname{Lip}_{Q, p, 0}(\Omega)$ using extension by 0 , and suppose the local Sobolev property of order $p$ in Definition 3.16 holds. Assume the cutoff property of some order $s \geq p \sigma^{\prime}$ is true for $\mu$, with $\sigma$ as in (3-31), and that (3-33) holds for $\operatorname{Lip}_{Q, p, 0}\left(\Omega^{\prime}\right)$ with $t=s / p$. Then, for every sequence $\left\{\left(f_{k}, \vec{g}_{k}\right)\right\} \subset W_{v, \mu, 0}^{1, p}\left(\Omega^{\prime}, Q\right)$ which is bounded in $W_{\nu, \mu}^{1, p}\left(\Omega^{\prime}, Q\right)$ norm, there is a subsequence $\left\{f_{k_{i}}\right\}$ of $\left\{f_{k}\right\}$ and a function $f \in L_{w}^{p \sigma}\left(\Omega^{\prime}\right)$ such that $f_{k_{i}} \rightarrow f$ pointwise a.e.-w in $\Omega^{\prime}$ and in $L_{w}^{q}\left(\Omega^{\prime}\right)$ norm, $1 \leq q<p \sigma$.

The full force of the local Sobolev estimate in Definition 3.16 is not needed to prove Theorem 3.24. In fact, it is enough to assume that (3-31) holds only for balls centered in the fixed compact set $\bar{\Omega}^{\prime}$.

The proof of Theorem 3.24 is like that of Theorem 3.20, working with the set $\Omega^{\prime}$ that occurs in the hypotheses of Theorem 3.24. However, now (3-34) in the conclusion of Lemma 3.23 (with $\mathscr{H}=\operatorname{Lip}_{Q, p, 0}\left(\Omega^{\prime}\right)$ ) remains valid if $\Omega^{\prime}$ is replaced on the left side by $\Omega$ since every $f \in \operatorname{Lip}_{Q, p, 0}\left(\Omega^{\prime}\right)$ vanishes on $\Omega \backslash \Omega^{\prime}$. The resulting estimate serves as a replacement for (3-38), so it is not necessary to demand that the $E_{l}^{\epsilon}$ are subsets of a compact set $\bar{\Omega}^{\prime \prime} \subset \Omega$. Hence (3-12) is no longer required. Finally, the Poincaré estimate extends as usual to $W_{v, \mu, 0}^{1, p}\left(\Omega^{\prime}, Q\right)$ (the closure of $\operatorname{Lip}_{Q, p, 0}\left(\Omega^{\prime}\right)$ ), and due to support considerations, the $E_{l}^{\epsilon}$ can be restricted to subsets of $\Omega^{\prime}$ by replacing $E_{l}^{\epsilon}$ by $E_{l}^{\epsilon} \cap \Omega^{\prime}$; this guarantees $w\left(E_{l}^{\epsilon}\right)<\infty$ since $w$ is locally finite by hypothesis.

Recalling the comments immediately after Definition 3.18 and in Remark 3.19, we obtain a useful special case of Theorem 3.24:

Corollary 3.25. Let the assumptions of Section $3 A$ hold, $\Omega$ and $Q$ be bounded, $w=$ $v=\mu$, and (3-12) be true. Let $\Omega^{\prime}$ be an open set with $\bar{\Omega}^{\prime} \subset \Omega$. Fix $p \in[1, \infty)$ and suppose the Poincaré property of order $p$ in Definition 3.5 holds for $\operatorname{Lip}_{Q, p, 0}\left(\Omega^{\prime}\right)$ and the local Sobolev property of order $p$ in Definition 3.16 holds. Then, for every $\left\{\left(f_{k}, \vec{g}_{k}\right)\right\} \subset W_{v, \mu, 0}^{1, p}\left(\Omega^{\prime}, Q\right)$ which is bounded in $W_{\nu, \mu}^{1, p}(\Omega, Q)$ norm, there is a subsequence $\left\{f_{k_{i}}\right\}$ of $\left\{f_{k}\right\}$ and a function $f \in L_{w}^{p \sigma}\left(\Omega^{\prime}\right)$ such that $f_{k_{i}} \rightarrow f$ pointwise a.e.-w in $\Omega^{\prime}$ and in $L_{w}^{q}\left(\Omega^{\prime}\right)$ norm, $1 \leq q<p \sigma$.

In the case where $p=2$ and all measures are Lebesgue measure, Corollary 3.25 is used in [Rodney 2007; 2012] to show the existence of weak solutions to Dirichlet problems for some linear subelliptic equations. It is also used in [Rodney 2010] to derive the following global Sobolev inequality from the local estimate (3-32), where $\Omega^{\prime}$ is open and $\bar{\Omega}^{\prime} \subset \Omega$ :

$$
\|f\|_{L^{2 \sigma}\left(\Omega^{\prime}\right)} \leq C\left(\int_{\Omega^{\prime}}|\sqrt{Q} \nabla f|^{2} d x\right)^{1 / 2} .
$$




\section{Precompact subsets of $L^{N}$ in a quasimetric space}

In this section, we consider the situation of an open set $\Omega$ in a topological space $X$ when $X$ is also endowed with a quasimetric $d$. As there is no easy way to define Sobolev spaces on general quasimetric spaces, this section concentrates on establishing a simple criterion not directly related to Sobolev spaces ensuring that bounded subsets of $L_{w}^{N}(\Omega)$ are precompact in $L_{w}^{q}(\Omega)$ when $1 \leq q<N \leq \infty$.

We begin by further describing the setting for our result. The topology on $X$ is expressed in terms of a fixed collection $\mathcal{T}$ of subsets of $X$ which may not be related to the quasimetric $d$. Thus when we say that a set $O \subset X$ is open, we mean that $0 \in \mathcal{T}$. Given an open $\Omega$, we assume the following:

(i) $\forall x \in X$ and $r>0$, the $d$-ball $B_{r}(x)=\{y \in X: d(x, y)<r\}$ is a Borel set.

(ii) $\forall x \in X$ and $r>0$, there is an open set 0 such that $x \in \mathcal{O} \subset B_{r}(x)$.

(iii) If $X \neq \Omega$, then $\forall x \in \Omega, d\left(x, \Omega^{c}\right)=\inf \left\{d(x, y): y \in \Omega^{c}\right\}>0$.

Property (ii) serves as a substitute for (3-2).

Unlike the situation in Section 3, $d$-balls centered in $\Omega$ may not be subsets of $\Omega$ unless $X=\Omega$. However, we note the following fact.

Remark 4.1. Properties (ii) and (iii) guarantee that for any compact set $K \subset \Omega$, there exists $\varepsilon(K)>0$ such that $B_{r}(x) \subset \Omega$ if $x \in K$ and $r<\varepsilon(K)$. In fact, first note that for any $x \in \Omega$, (iii) implies that the $d$-ball $B(x)$ with center $x$ and radius $r_{x}=d\left(x, \Omega^{c}\right) /(2 \kappa)$ lies in $\Omega$. If $K$ is a compact set in $\Omega$, (ii) shows that $K$ can be covered by a finite number of such balls $\left\{B\left(x_{i}\right)\right\}$. With $\varepsilon(K)$ chosen to be a suitably small multiple (depending on $\kappa$ ) of $\min \left\{r_{x_{i}}\right\}$, the remark then follows easily from the swallowing property of $d$-balls.

Further, we assume that $(\Omega, d)$ satisfies the local geometric doubling condition in Definition 3.3, that is, for each compact set $K \subset \Omega$, there exists $\delta^{\prime}(K)>0$ such that, for all $x \in K$ and all $0<r^{\prime}<r<\delta^{\prime}(K)$, the number of disjoint $d$-balls of common radius $r^{\prime}$ contained in $B_{r}(x)$ is at most a constant $\mathscr{C}_{r / r^{\prime}}$ depending on $r / r^{\prime}$ but not on $K$. We will choose $\delta^{\prime}(K) \leq \varepsilon(K)$.

With this framework in force, we now state the main result of the section.

Theorem 4.2. Let $\Omega \subset X$ be as above, and let $w$ be a finite Borel measure on $\Omega$ such that, given any $\epsilon>0$, there is a compact set $K \subset \Omega$ with $w(\Omega \backslash K)<\epsilon$. Let $1 \leq p<\infty$ and $1<N \leq \infty$, and suppose $\mathscr{Y} \subset L_{w}^{N}(\Omega)$ has the property that, for any compact set $K \subset \Omega$, there exists $\delta_{K}>0$ such that

$$
\left\|f-f_{B, w}\right\|_{L_{w}^{p}(B)} \leq b(f, B) \quad \text { if } f \in \mathscr{Y} \text { and } B=B_{r}(x), x \in K, 0<r<\delta_{K},
$$

where $b(f, B)$ is a nonnegative ball set function. Furthermore, suppose there is a constant $c_{0} \geq 1$ such that for every $\epsilon>0$ and every compact set $K \subset \Omega$, there exists 
$\tilde{\delta}_{\epsilon, K}>0$ such that

$$
\sum_{B \in \mathscr{F}} b(f, B)^{p} \leq \epsilon^{p} \quad \text { for all } f \in \mathscr{Y}
$$

for every finite family $\mathscr{F}=\{B\}$ of $d$-balls centered in $K$ with common radius less than $\tilde{\delta}_{\epsilon, K}$ for which $\left\{c_{0} B\right\}$ is a pairwise disjoint family of subsets of $\Omega$. Then any sequence in $\mathscr{Y}$ that is bounded in $L_{w}^{N}(\Omega)$ has a subsequence that converges in $L_{w}^{q}(\Omega)$ for $1 \leq q<N$ to a function in $L_{w}^{N}(\Omega)$.

Proof. Let $\epsilon>0$ and choose a compact set $K \subset \Omega$ with $w(\Omega \backslash K)<\epsilon$. Next, for $c_{0} \geq 1$, as in the proof of Lemma 3.12, there is a positive constant $r=r\left(\epsilon, K, c_{0}\right)<$ $\min \left\{\delta_{K}, \tilde{\delta}_{\epsilon, K}, \delta^{\prime}(K), \varepsilon(K) /\left(\gamma c_{0}\right)\right\}$ (see (4-1),(4-2), Definition 3.3 and Remark 4.1), where $\gamma=\kappa+2 \kappa^{2}$ with $\kappa$ as in (3-1), and a finite family $\left\{B_{r}\left(y_{k}\right)\right\}_{k}$ of $d$-balls centered in $K$ satisfying $K \subset \bigcup_{k} B_{r}\left(y_{k}\right)$ and whose dilates $\left\{B_{c_{0} r}\left(y_{k}\right)\right\}_{k}$ lie in $\Omega$ and have the bounded intercept property (with intercept constant $M$ independent of $\epsilon$ ). Since $\left\{B_{c_{0} r}\left(y_{k}\right)\right\}_{k}$ has bounded intercepts with bound $M$, it can be written as the union of at most $M$ families of disjoint $d$-balls; see, for example, the proof of [Chua and Wheeden 2008, Lemma 2.5]. By (4-2), we conclude that

$$
\sum_{k} b\left(f, B_{r}\left(y_{k}\right)\right)^{p} \leq M \epsilon^{p} .
$$

Theorem 4.2 follows then immediately from Theorem 1.2; see also Remark 1.3(1).

As an application of Theorem 4.2 we present a version of [Hajłasz and Koskela 2000, Theorem 8.1] in the case $p \geq 1$. Our version improves the one in [Hajłasz and Koskela 2000] by allowing two different measures and by relaxing the assumptions made about embedding and doubling. Furthermore, while the analogue in [Hajłasz and Koskela 2000] of our (4-3) uses only the $L_{w}^{1}(B)$ norm on the left side, it automatically self-improves to the $L_{w}^{p}(B)$ norm due to the doubling assumption, with a further fixed enlargement of the ball $c_{0} B$ on the right side; see, for example, [Hajłasz and Koskela 2000, Theorem 5.1].

Corollary 4.3. Let $X, d, \Omega, w$ be as above, and let $\mu$ be a Borel measure on $\Omega$. Fix $1 \leq p<\infty, 1<N \leq \infty$, and $c_{0} \geq 1$. Consider a sequence of pairs

$$
\left\{\left(f_{i}, g_{i}\right)\right\} \subset L_{w}^{N}(\Omega) \times L_{\mu}^{p}(\Omega)
$$

such that, for any compact set $K \subset \Omega$, there exists $\bar{\delta}_{K}>0$ with

$$
\left\|f_{i}-\left(f_{i}\right)_{B, w}\right\|_{L_{w}^{p}(B)} \leq a_{*}(B)\left\|g_{i}\right\|_{L_{\mu}^{p}\left(c_{0} B\right)}
$$

for all $i$ and all $d$-balls $B$ centered in $K$ with $c_{0} B \subset \Omega$ and $r(B)<\bar{\delta}_{K}$, where 
$a_{*}(B)$ is a nonnegative ball set function satisfying

$$
\limsup _{r \rightarrow 0}\left\{\sup _{y \in K} a_{*}\left(B_{r}(y)\right)\right\}=0 .
$$

Then if $\left\{f_{i}\right\}$ and $\left\{g_{i}\right\}$ are bounded in $L_{w}^{N}(\Omega)$ and $L_{\mu}^{p}(\Omega)$, respectively, $\left\{f_{i}\right\}$ has a subsequence converging in $L_{w}^{q}(\Omega)$ for $1 \leq q<N$ to a function belonging to $L_{w}^{N}(\Omega)$.

Proof. Given $\epsilon>0$ and compact set $K \subset \Omega$, use (4-4) to choose $r_{0}>0$ such that $a_{*}\left(B_{r}\right)<\epsilon / \beta$ for any $d$-ball $B_{r}$ centered in $K$ with $r<r_{0}$, where $\beta=$ $\sup _{i}\left\|g_{i}\right\|_{L_{\mu}^{p}(\Omega)}<\infty$. In Theorem 4.2, choose $\mathscr{Y}=\left\{f_{i}\right\}, \delta_{K}=\bar{\delta}_{K}, b\left(f_{i}, B\right)=$ $a_{*}(B)\left\|g_{i}\right\|_{L_{\mu}^{p}\left(c_{0} B\right)}$, and

$$
\tilde{\delta}_{\epsilon, K}=\min \left\{\bar{\delta}_{K}, \delta^{\prime}(K), r_{0}, \varepsilon(K) / c_{0}\right\} .
$$

If $B$ is a $d$-ball with center in $K$ and $r(B)<\tilde{\delta}_{\epsilon, K}$, then $c_{0} B \subset \Omega$. Hence

$$
\sum_{B \in \mathscr{F}}\left(a_{*}(B)\left\|g_{i}\right\|_{L_{\mu}^{p}\left(c_{0} B\right)}\right)^{p} \leq \epsilon^{p}\left\|g_{i}\right\|_{L_{\mu}^{p}(\Omega)}^{p} / \beta^{p} \leq \epsilon^{p}
$$

for every $\mathscr{F}$ as in Theorem 4.2. The conclusion now follows from Theorem 4.2.

Remark 4.4. (1) The $g_{i}$ in (4-3) are usually the modulus of a fixed derivative of the corresponding $f_{i}$, such as $\left|\nabla f_{i}\right|$ when $X$ is a Riemannian manifold. More generally, $g_{i}$ may be the upper gradient of $f_{i}$ (see [Heinonen 2001] for the definition).

(2) Theorem 4.2 can also be used to obtain an extension of Theorem 2.4 to $s$-John domains in quasimetric spaces; see [Chua and Wheeden 2011, Theorem 1.6].

\section{Appendix}

We briefly consider analogues of Theorem 3.14, Corollary 3.15, and Theorem 3.20 without assuming $w \ll v$, but adding the assumption that $\mathscr{H}$ is linear. In this case, (3-27) can be extended by continuity to obtain a bounded linear map from $\overline{\mathscr{H}}$ into $L_{w}^{p \sigma}(\Omega)$. Here, as always, $\overline{\mathscr{H}}$ denotes the closure of $\{(f, \nabla f): f \in \mathscr{H}\}$ in $W_{\nu, \mu}^{1, p}(\Omega, Q)$. However, when $w \ll v$ fails, there is no natural way to obtain the extension for every $(f, \vec{g}) \in \overline{\mathscr{H}}$ keeping the same $f$ on the left side. In fact, let $(f, \vec{g}) \in \overline{\mathscr{H}}$ and choose $\left\{f_{j}\right\} \subset \mathscr{H}$ with $\left(f_{j}, \nabla f_{j}\right) \rightarrow(f, \vec{g})$ in $W_{\nu, \mu}^{1, p}(\Omega, Q)$. Linearity of $\mathscr{H}$ allows us to apply (3-27) to differences of the $f_{j}$ and conclude that $\left\{f_{j}\right\}$ is a Cauchy sequence in $L_{w}^{p \sigma}(\Omega)$. Therefore $f_{j} \rightarrow f^{*}$ in $L_{w}^{p \sigma}(\Omega)$ for some $f^{*} \in L_{w}^{p \sigma}(\Omega)$, and

$$
\left\|f^{*}\right\|_{L_{w}^{p \sigma}(\Omega)} \leq C\|(f, \vec{g})\|_{W_{\nu, \mu}^{1, p}(\Omega, Q)} \quad \text { if }(f, \vec{g}) \in \overline{\mathscr{H}} .
$$


The function $f^{*}$ is determined by $(f, \vec{g})$, that is, $f^{*}$ is independent of the particular sequence $\left\{f_{j}\right\} \subset \mathscr{H}$ above. Indeed, if $\left\{\tilde{f}_{j}\right\}$ is another sequence in $\mathscr{H}$ with $\left(\tilde{f}_{j}, \nabla \tilde{f}_{j}\right) \rightarrow(f, \vec{g})$ in $W_{\nu, \mu}^{1, p}(\Omega, Q)$, and if $\tilde{f}_{j} \rightarrow \tilde{f}^{*}$ in $L_{w}^{p \sigma}(\Omega)$, then, by (3-27) and linearity of $\mathscr{H}$,

$$
\left\|\tilde{f}_{j}-f_{j}\right\|_{L_{w}^{p \sigma}(\Omega)} \leq C\left\|\left(\tilde{f}_{j}-f_{j}, \nabla \tilde{f}_{j}-\nabla f_{j}\right)\right\|_{W_{\nu, \mu}^{1, p}(\Omega, Q)} \rightarrow 0 .
$$

Consequently $\left\|\tilde{f}^{*}-f^{*}\right\|_{L_{w}^{p \sigma}(\Omega)}=0$. Thus $(f, \vec{g})$ determines $f^{*}$ uniquely as an element of $L_{w}^{p \sigma}(\Omega)$. Define a mapping

$$
\text { (A-1) } \quad T: \overline{\mathscr{H}} \rightarrow L_{w}^{p \sigma}(\Omega) \text { by setting } T(f, \vec{g})=f^{*} \text {. }
$$

Note that $\overline{\mathscr{H}}$ is a linear set in $W_{\nu, \mu}^{1, p}(\Omega, Q)$ since $\mathscr{H}$ is linear, and that $T$ is a bounded linear map from $\overline{\mathscr{H}}$ into $L_{w}^{p \sigma}(\Omega)$. Also note that $T$ satisfies $T(f, \nabla f)=f$ when restricted to those $(f, \nabla f)$ with $f \in \mathcal{H}$. Furthermore, if $w \ll v$, then $T(f, \vec{g})=f$ for all $(f, \vec{g}) \in \overline{\mathscr{H}}$, that is, $f^{*}=f$ a.e.- $w$ for all $(f, \vec{g}) \in \overline{\mathscr{H}}$. This follows since $f_{j} \rightarrow f$ in $L_{v}^{p}(\Omega)$ norm and $f_{j} \rightarrow f^{*}$ in $L_{w}^{p \sigma}(\Omega)$ norm. In this appendix, where it is not assumed that $w \ll v, f^{*}$ plays a main role. One can find a function $h$ such that $h=f^{*}$ a.e.- $w$ and $h=f$ a.e.- $v$, but as this fact is not needed, we omit its proof.

An analogue of Theorem 3.14 is given in the next result.

Theorem A.1. Let all the assumptions of Theorem 3.14 hold except that now the set $\mathscr{H}$ is linear and we do not assume $w \ll v$. Then the map $T: \overline{\mathcal{H}} \rightarrow L_{w}^{q}(\Omega)$ defined in (A-1) is compact if $1 \leq q<p \sigma$. Equivalently, if $\left\{\left(f_{k}, \overrightarrow{g_{k}}\right)\right\}$ is a sequence in $\overline{\mathcal{H}}$ with $\sup _{k}\left\|\left(f_{k}, \overrightarrow{g_{k}}\right)\right\|_{W_{\nu, \mu}^{1, p}}(\Omega, Q)<\infty$, then $\left\{f_{k}^{*}\right\}$ has a subsequence which converges in $L_{w}^{q}(\Omega)$ for $1 \leq \underset{q}{ }<p \sigma$, where $f_{k}^{*}=T\left(f_{k}, \vec{g}_{k}\right)$. Moreover, the limit of the subsequence belongs to $L_{w}^{p \sigma}(\Omega)$.

Proof. Let $\mathscr{H}$ satisfy the hypothesis of the theorem and let $\left\{\left(f_{k}, \vec{g}_{k}\right)\right\} \subset \overline{\mathscr{H}}$ be bounded in $W_{\nu, \mu}^{1, p}(\Omega, Q)$. For each $k$, choose $h_{k} \in \mathscr{H}$ such that

$$
\left\|\left(f_{k}, \vec{g}_{k}\right)-\left(h_{k}, \nabla h_{k}\right)\right\|_{W_{v, \mu}^{1, p}(\Omega, Q)} \leq 2^{-k} \text {. }
$$

Set $\mathscr{H}_{1}=\left\{h_{k}\right\}_{k} \subset \mathscr{H}$. Then $\left\{\left(h_{k}, \nabla h_{k}\right): h_{k} \in \mathscr{H}_{1}\right\}$ is bounded in $W_{\nu, \mu}^{1, p}(\Omega, Q)$. Furthermore, (3-27) implies a version of (3-14), namely,

$$
\sup _{f \in \mathscr{H}_{1}}\left\{\|f\|_{L_{w}^{p \sigma}(\Omega)}+\|(f, \nabla f)\|_{W_{\nu, \mu}^{1, p}(\Omega, Q)}\right\}<\infty .
$$

Theorem 3.7 now applies to $\mathscr{H}_{1}$ with $N=p \sigma$ and gives that any sequence in $\widehat{\mathscr{H}_{1}}$ has a subsequence which converges in $L_{w}^{q}(\Omega)$ norm for $1 \leq q<p \sigma$ to a function belonging to $L_{w}^{p \sigma}(\Omega)$. The sequence $\left\{h_{k}\right\}$ lies in $\widehat{\mathscr{H}_{1}}$, as is easily seen by considering, for each fixed $k$, the constant sequence $\left\{f^{j}\right\}$ defined by $f^{j}=h_{k}$ for 
all $j$. We conclude that $\left\{h_{k}\right\}$ has a subsequence $\left\{h_{k_{l}}\right\}$ converging in $L_{w}^{q}(\Omega)$ norm for $1 \leq q<p \sigma$ to a function $h \in L_{w}^{p \sigma}(\Omega)$. By linearity and boundedness of $T$ from $\overline{\mathscr{H}}$ to $L_{w}^{p \sigma}(\Omega)$ together with (A-2), we have (writing $f_{k}^{*}=T\left(f_{k}, \overrightarrow{g_{k}}\right)$ )

$$
\left\|f_{k}^{*}-h_{k}\right\|_{L_{w}^{p \sigma}(\Omega)}=\left\|T\left(f_{k}, \vec{g}_{k}\right)-T\left(h_{k}, \nabla h_{k}\right)\right\|_{L_{w}^{p \sigma}(\Omega)} \leq C 2^{-k} \rightarrow 0 .
$$

Restricting $k$ to $\left\{k_{l}\right\}$ and using $w(\Omega)<\infty$, we conclude that $\left\{f_{k_{l}}^{*}\right\}$ also converges to $h$ in $L_{w}^{q}(\Omega)$ for $1 \leq q<p \sigma$, which completes the proof.

Setting $\mathscr{H}=\operatorname{Lip}_{Q, p}(\Omega)$ in Theorem A.1 gives an analogue of Corollary 3.15.

Corollary A.2. Let the hypotheses of Theorem A.1 hold for $\mathscr{H}=\operatorname{Lip}_{Q, p}(\Omega)$. Then the map $T$ defined by (A-1) is a compact map of $W_{\nu, \mu}^{1, p}(\Omega, Q)$ into $L_{w}^{q}(\Omega)$ for $1 \leq$ $q<p \sigma$, that is, if $\left\{\left(f_{k}, \vec{g}_{k}\right)\right\} \subset W_{\nu, \mu}^{1, p}(\Omega, Q)$ and $\sup _{k}\left\|\left(f_{k}, \vec{g}_{k}\right)\right\|_{W_{\nu, \mu}^{1, p}(\Omega, Q)}<\infty$, then $\left\{f_{k}^{*}\right\}$ has a subsequence which converges in $L_{w}^{q}(\Omega)$ for $1 \leq q<p \sigma$, where $f_{k}^{*}=T\left(f_{k}, \overrightarrow{g_{k}}\right)$. Moreover, the limit of the subsequence belongs to $L_{w}^{p \sigma}(\Omega)$.

Theorem 3.20 also has an analogue without assuming $w \ll v$ provided $\mathscr{H}$ is linear, and in this instance (3-27) is not required: the subsequence $\left\{f_{k_{i}}\right\}$ of $\left\{f_{k}\right\}$ in the conclusion is then replaced by a subsequence of $\left\{f_{k}^{*}\right\}$, where $f_{k}^{*}$ is constructed as above but now using bounded open $\Omega^{\prime}$ whose closures increase to $\Omega$. Now $f^{*}$ arises when (3-38) is extended to $\overline{\mathscr{H}}$, namely, instead of (3-39), we obtain

$$
\left\|f^{*}\right\|_{L_{w}^{p \sigma}\left(\Omega^{\prime \prime}\right)} \leq C\left(\Omega^{\prime \prime}\right)\|(f, \vec{g})\|_{W_{\nu, \mu}^{1, p}(\Omega, Q)} \quad \text { if }(f, \vec{g}) \in \overline{\mathscr{H}}
$$

where $f^{*}$ is constructed for a pair $(f, \vec{g}) \in \overline{\mathscr{H}}$ by using linearity of $\mathscr{H}$ and (3-38) for a particular $\left(\Omega^{\prime}, \Omega^{\prime \prime}\right)$. It is easy to see that $f^{*} \in L_{w, \text { loc }}^{p \sigma}(\Omega)$ by letting $\Omega^{\prime} \nearrow \Omega$. The Poincaré inequality analogous to (3-40) is

$$
\left(\int_{B_{r}(y)}\left|f^{*}-f_{B_{r}(y), w}^{*}\right|^{p} d w\right)^{1 / p} \leq \epsilon\|(f, \vec{g})\|_{W_{\nu, \mu}^{1, p}\left(B_{c_{0} r}(y), Q\right)} \quad \text { if }(f, \vec{g}) \in \overline{\mathscr{H}},
$$

obtained by extending (3-8) from $\mathscr{H}$ to $\overline{\mathcal{H}}$. Further details are omitted.

\section{References}

[Adams and Fournier 2003] R. A. Adams and J. J. F. Fournier, Sobolev spaces, 2nd ed., Pure and Applied Mathematics 140, Academic Press, New York, 2003. MR 2009e:46025 Zbl 1098.46001

[Aubin 1976] T. Aubin, "Équations différentielles non linéaires et problème de Yamabe concernant la courbure scalaire", J. Math. Pures Appl. (9) 55:3 (1976), 269-296. MR 55 \#4288 Zbl 0336.53033

[Chua 1995] S.-K. Chua, "Weighted Sobolev interpolation inequalities on certain domains", J. London Math. Soc. (2) 51:3 (1995), 532-544. MR 96d:46033 Zbl 0845.26008

[Chua and Wheeden 2008] S.-K. Chua and R. L. Wheeden, "Self-improving properties of inequalities of Poincaré type on measure spaces and applications", J. Funct. Anal. 255:11 (2008), 2977-3007. MR 2010f:46050 Zbl 1172.46020 
[Chua and Wheeden 2011] S.-K. Chua and R. L. Wheeden, "Self-improving properties of inequalities of Poincaré type on s-John domains", Pacific J. Math. 250:1 (2011), 67-108. MR 2012c:46068 Zbl 1214.26014

[Fabes et al. 1982] E. B. Fabes, C. E. Kenig, and R. P. Serapioni, "The local regularity of solutions of degenerate elliptic equations", Comm. Partial Differential Equations 7:1 (1982), 77-116. MR 84i:35070 Zbl 0498.35042

[Franchi et al. 1997] B. Franchi, R. Serapioni, and F. Serra Cassano, "Approximation and imbedding theorems for weighted Sobolev spaces associated with Lipschitz continuous vector fields", Boll. Un. Mat. Ital. B (7) 11:1 (1997), 83-117. MR 98c:46062 Zbl 0952.49010

[Gilbarg and Trudinger 1997] D. Gilbarg and N. S. Trudinger, Elliptic partial differential equations of second order, 3rd ed., Grundlehren der mathematischen Wissenschaften 224, Springer, Berlin, 1997. MR 2001k:35004 Zbl 1042.35002

[Hajłasz 1993] P. Hajłasz, "Note on Meyers-Serrin's theorem”, Exposition. Math. 11:4 (1993), 377-379. MR 94e:46060 Zbl 0799.46042

[Hajłasz and Koskela 1998] P. Hajłasz and P. Koskela, "Isoperimetric inequalities and imbedding theorems in irregular domains", J. London Math. Soc. (2) 58:2 (1998), 425-450. MR 99m:46079 Zbl 0922.46034

[Hajłasz and Koskela 2000] P. Hajłasz and P. Koskela, Sobolev met Poincaré, Mem. Amer. Math. Soc. 688, American Mathematical Society, 2000. MR 2000j:46063 Zbl 0952.46022

[Heinonen 2001] J. Heinonen, Lectures on analysis on metric spaces, Springer, New York, 2001. MR 2002c:30028 Zbl 0985.46008

[Hytönen and Martikainen 2012] T. Hytönen and H. Martikainen, "Non-homogeneous Tb theorem and random dyadic cubes on metric measure spaces", J. Geom. Anal. 22:4 (2012), 1071-1107. MR 2965363 Zbl 06124339

[Kilpeläinen and Malý 2000] T. Kilpeläinen and J. Malý, "Sobolev inequalities on sets with irregular boundaries", Z. Anal. Anwendungen 19:2 (2000), 369-380. MR 2001g:46075 Zbl 0959.46020

[Monticelli et al. 2012] D. D. Monticelli, S. Rodney, and R. L. Wheeden, "Boundedness of weak solutions of degenerate quasilinear equations with rough coefficients", Differential Integral Equations 25:1-2 (2012), 143-200. MR 2906551 Zbl 1249.35117

[Rios et al. 2013] C. Rios, E. T. Sawyer, and R. L. Wheeden, "Hypoellipticity for infinitely degenerate quasilinear equations and the Dirichlet problem", J. d'Analyse Math. 119 (2013), 1-62. MR 3043146 Zbl 06186919

[Rodney 2007] S. W. Rodney, Existence of weak solutions to subelliptic partial differential equations in divergence form and the necessity of the Sobolev and Poincare inequalities, Ph.D. thesis, McMaster University, 2007, available at http://search.proquest.com/docview/304819194. MR 2711279

[Rodney 2010] S. Rodney, "A degenerate Sobolev inequality for a large open set in a homogeneous space”, Trans. Amer. Math. Soc. 362:2 (2010), 673-685. MR 2011f:35055 Zbl 1190.35010

[Rodney 2012] S. Rodney, "Existence of weak solutions of linear subelliptic Dirichlet problems with rough coefficients", Canad. J. Math. 64:6 (2012), 1395-1414. MR 2994671 Zbl 06111146

[Rudin 1987] W. Rudin, Real and complex analysis, 3rd ed., McGraw-Hill, New York, 1987. MR 88k:00002 Zbl 0925.00005

[Sawyer and Wheeden 2006] E. T. Sawyer and R. L. Wheeden, Hölder continuity of weak solutions to subelliptic equations with rough coefficients, Mem. Amer. Math. Soc. 847, American Mathematical Society, 2006. MR 2007f:35037 Zbl 1096.35031 
[Sawyer and Wheeden 2010] E. T. Sawyer and R. L. Wheeden, "Degenerate Sobolev spaces and regularity of subelliptic equations", Trans. Amer. Math. Soc. 362:4 (2010), 1869-1906. MR 2010m:35077 Zbl 1191.35085

[Schoen 1984] R. Schoen, "Conformal deformation of a Riemannian metric to constant scalar curvature”, J. Differential Geom. 20:2 (1984), 479-495. MR 86i:58137 Zbl 0576.53028

[Trudinger 1968] N. S. Trudinger, "Remarks concerning the conformal deformation of Riemannian structures on compact manifolds", Ann. Scuola Norm. Sup. Pisa (3) 22 (1968), 265-274. MR 39 \#2093 Zbl 0159.23801

[Turesson 2000] B. O. Turesson, Nonlinear potential theory and weighted Sobolev spaces, Lecture Notes in Mathematics 1736, Springer, Berlin, 2000. MR 2002f:31027 Zbl 0949.31006

[Yamabe 1960] H. Yamabe, "On a deformation of Riemannian structures on compact manifolds", Osaka Math. J. 12 (1960), 21-37. MR 23 \#A2847 Zbl 0096.37201

Received July 25, 2012.

SENG-KeE CHUA

DEPARTMENT OF MATHEMATICS

NATIONAL UNIVERSITY OF SINGAPORE

10, LOWER KENT RIDGE ROAD

SINGAPORE 119076

SINGAPORE

matcsk@nus.edu.sg

\section{SCOTT RODNEY}

Department of Mathematics, Physics, and Geology

CAPE BRETON UNIVERSITY

P.O. BOX 5300, 1250 GRAND LAKE ROAD

SYDNEY, NS B1P 6L2

CANADA

scott_rodney@cbu.ca

RICHARD L. WHEEDEN

DEPARTMENT OF MATHEMATICS

RUTGERS UNIVERSITY

110 FRELINGHUYSEN ROAD

PISCATAWAY, NJ 08854

UNITED STATES

wheeden@math.rutgers.edu 


\title{
PACIFIC JOURNAL OF MATHEMATICS
}

\author{
msp.org/pjm
}

Founded in 1951 by E. F. Beckenbach (1906-1982) and F. Wolf (1904-1989)

\section{EDITORS}

V. S. Varadarajan (Managing Editor)

Department of Mathematics

University of California

Los Angeles, CA 90095-1555

pacific@math.ucla.edu

Paul Balmer

Department of Mathematics

University of California

Los Angeles, CA 90095-1555

balmer@math.ucla.edu

Daryl Cooper

Department of Mathematics

University of California

Santa Barbara, CA 93106-3080 cooper@math.ucsb.edu

Jiang-Hua $\mathrm{Lu}$

Department of Mathematics

Pokfulam Rd., Hong Kong jhlu@maths.hku.hk
The University of Hong Kong

Don Blasius

Department of Mathematics University of California

Los Angeles, CA 90095-1555

blasius@math.ucla.edu

Robert Finn

Department of Mathematics Stanford University

Stanford, CA 94305-2125

finn@math.stanford.edu

Sorin Popa

Department of Mathematics

University of California

Los Angeles, CA 90095-1555 popa@math.ucla.edu

Paul Yang

Department of Mathematics Princeton University

Princeton NJ 08544-1000

yang@math.princeton.edu

\section{PRODUCTION}

Silvio Levy, Scientific Editor, production@msp.org

\section{SUPPORTING INSTITUTIONS}

ACADEMIA SINICA, TAIPEI

CALIFORNIA INST. OF TECHNOLOGY

INST. DE MATEMÁTICA PURA E APLICADA

KEIO UNIVERSITY

MATH. SCIENCES RESEARCH INSTITUTE

NEW MEXICO STATE UNIV.

OREGON STATE UNIV.

\author{
STANFORD UNIVERSITY \\ UNIV. OF BRITISH COLUMBIA \\ UNIV. OF CALIFORNIA, BERKELEY \\ UNIV. OF CALIFORNIA, DAVIS \\ UNIV. OF CALIFORNIA, LOS ANGELES \\ UNIV. OF CALIFORNIA, RIVERSIDE \\ UNIV. OF CALIFORNIA, SAN DIEGO \\ UNIV. OF CALIF., SANTA BARBARA
}

\author{
Vyjayanthi Chari \\ Department of Mathematics \\ University of California \\ Riverside, CA 92521-0135 \\ chari@math.ucr.edu \\ Kefeng Liu \\ Department of Mathematics \\ University of California \\ Los Angeles, CA 90095-1555 \\ liu@math.ucla.edu \\ Jie Qing \\ Department of Mathematics \\ University of California \\ Santa Cruz, CA 95064 \\ qing@cats.ucsc.edu
}

These supporting institutions contribute to the cost of publication of this Journal, but they are not owners or publishers and have no responsibility for its contents or policies.

See inside back cover or msp.org/pjm for submission instructions.

The subscription price for 2013 is US \$400/year for the electronic version, and \$485/year for print and electronic.

Subscriptions, requests for back issues and changes of subscribers address should be sent to Pacific Journal of Mathematics, P.O. Box 4163, Berkeley, CA 94704-0163, U.S.A. The Pacific Journal of Mathematics is indexed by Mathematical Reviews, Zentralblatt MATH, PASCAL CNRS Index, Referativnyi Zhurnal, Current Mathematical Publications and the Science Citation Index.

The Pacific Journal of Mathematics (ISSN 0030-8730) at the University of California, c/o Department of Mathematics, 798 Evans Hall \#3840, Berkeley, CA 94720-3840, is published twelve times a year. Periodical rate postage paid at Berkeley, CA 94704, and additional mailing offices. POSTMASTER: send address changes to Pacific Journal of Mathematics, P.O. Box 4163, Berkeley, CA 94704-0163.

PJM peer review and production are managed by EditFLOW ${ }^{\circledR}$ from Mathematical Sciences Publishers.

\section{PUBLISHED BY}

mathematical sciences publishers

nonprofit scientific publishing

http://msp.org/

(C) 2013 Mathematical Sciences Publishers 


\section{PACIFIC JOURNAL OF MATHEMATICS}

Volume $265 \quad$ No. $1 \quad$ September 2013

Genus-two Goeritz groups of lens spaces

SANGBUM CHO

A compact embedding theorem for generalized Sobolev spaces

SENG-KeE ChUa, SCOTT Rodney and Richard L. WheEden

Partial integrability of almost complex structures and the existence of

solutions for quasilinear Cauchy-Riemann equations

CHONG-KYU HAN and JONG-DO PARK

An overdetermined problem in potential theory

DMITRY KHAVINSON, ERIK LUNDBERG and RAZVAN TEODORESCU

Quasisymmetric homeomorphisms on reducible Carnot groups

XIANGDONG XIE

Capillarity and Archimedes' principle

JOHN MCCUAN and RAY TREINEN

Generalized eigenvalue problems of nonhomogeneous elliptic operators and their application

DUmitru Motreanu and Mieko TANaKa

Weighted Ricci curvature estimates for Hilbert and Funk geometries

SHIN-ICHI OHTA

On generalized weighted Hilbert matrices

EMMANUEL PREISSMANN and OLIVIER LÉVÊQUE

Unique prime decomposition results for factors coming from wreath product 221 groups

J. OWEN SizEMORE and ADAM WinCHESTER

On volume growth of gradient steady Ricci solitons

Guofang Wei and Peng Wu

Classification of moduli spaces of arrangements of nine projective lines

FEI YE 